This copy is made for your private study or for your research.

The Copyright Act 1994 prohibits the sale, letting for hire or copying of this copy. 
International Journal of Theoretical and Applied Finance

Vol. 23, No. 7 (2020) 2050048 (33 pages)

(C) World Scientific Publishing Company

DOI: 10.1142/S021902492050048X

\title{
APPROXIMATING THE GROWTH OPTIMAL PORTFOLIO AND STOCK PRICE BUBBLES
}

\author{
ECKHARD PLATEN* \\ School of Mathematical and \\ Physical Sciences and Finance Discipline Group \\ University of Technology Sydney \\ College of Business and Economics \\ Australian National University, Canberra \\ Department of Actuarial Science, University of Cape Town \\ eckhard.platen@uts.edu.au \\ RENATA RENDEK \\ Quantitative Finance Research Centre \\ University of Technology Sydney \\ renata.rendek@gmail.com
}

Received 5 August 2019

Revised 6 June 2020

Accepted 5 October 2020

Published 21 November 2020

\begin{abstract}
In practice, optimal portfolio construction for large stock markets has never been conclusively resolved because estimating the required means of returns with sufficient accuracy is a highly intractable task. By avoiding estimation, this paper approximates closely the growth optimal portfolio (GP) for the stocks of developed markets with a well-diversified, hierarchically weighted index (HWI). For stocks denominated in units of the HWI, their current value turns out to be strictly greater than their future expected values, which indicates the existence of stock price bubbles that could be systematically exploited for long-term asset management. It is shown that the HWI does not leave much room for significant performance improvements as proxy for the GP.
\end{abstract}

Keywords: Growth optimal portfolio; hierarchical diversification; strict supermartingale property; benchmark approach; stock price bubbles.

\section{Introduction}

The question, of how to construct, in practice, optimal portfolios for large stock markets, has been widely discussed in the literature but never been conclusively answered. Estimation-based attempts failed because it seems impossible to estimate with sufficient accuracy the means of stock returns, required for the construction

*Corresponding author. 
of theoretically optimal portfolios; see, e.g. DeMiguel et al. (2009). By overcoming this deadlock, the current paper makes the following three contributions:

First, it provides a solution to the long-standing problem of optimal portfolio construction and demonstrates this solution for stocks of the developed markets by approximating the respective growth optimal portfolio (GP) with a hierarchically weighted index (HWI).

Second, it shows empirically that the defining property of the GP, where stocks denominated in units of the GP have zero expected instantaneous returns, cannot be easily rejected for the HWI.

Third, it reveals the strict supermartingale property for stocks denominated in units of the HWI, where the current value is strictly greater than the expected future values, which indicates the existence of stock price bubbles.

Note that a strict local martingale is a strict supermartingale when positive. Under classical no-arbitrage assumptions, stocks denominated in units of the GP are assumed to be true martingales, which means that their current value is assumed to be the best forecast of their future values. For instance, by identifying the GP as the, so called, numeraire portfolio, Long (1990) derived the above martingale property for risk-neutral prices of contingent claims when these prices are denominated in units of the GP. Similarly, by using the notion of a, so-called, stochastic discount factor, which one may choose as the inverse of the discounted GP, Cochrane (2001) assumes that discounted securities multiplied by such a stochastic discount factor form true martingales. Thus, the third contribution provides strong evidence for a violation of crucial, widely used assumptions in financial economics. It hints at the existence of, so-called, asset bubbles, which have been theoretically anticipated and discussed, e.g. in Loewenstein \& Willard (2000a), Loewenstein \& Willard (2000b), Platen (2002), Heston et al. (2007) and Baldeaux et al. (2018). Furthermore, the work of Karatzas \& Kardaras (2007) shows that the no-unbounded-profit-withbounded-risk no arbitrage condition still holds, whereas the no-free-lunch-withvanishing-risk no arbitrage condition by Delbaen \& Schachermaver (1998) fails to hold.

\subsection{Optimal portfolios}

The GP is the expected logarithmic utility maximizing portfolio. It can be traced back to Kelly (1956) and became widely studied in the literature; see MacLean et al. (2011) for a collection of key papers and references. In this context, it is worth mentioning that we use the notions of a GP and a logarithmic utility maximizing portfolio interchangeably even that there exist subtle differences between these in the literature; see Karatzas \& Kardaras (2007). The GP can be employed as central building block for the construction of most optimal portfolios, including those at the efficient portfolio frontier and expected utility maximizing portfolios; see, e.g. Markowitz (1995) and Merton (1971). 
This paper shows that the GP is a very special optimal portfolio: The GP is the only optimal portfolio that can be approximated directly via diversification without involving estimation. Therefore, by approximating the GP for stocks of the developed markets one is gaining access to other optimal portfolios.

Building on Markowitz's modern portfolio theory, Sharpe (1964) introduced the capital asset pricing model (CAPM), which became generalized in a stream of literature. Empirical evidence shows that the market capitalization weighted index (MCI) may not yield the highest Sharpe ratio, as suggested by the CAPM; see, e.g. Harvey et al. (2014). The current paper confirms this stylized empirical fact by constructing a proxy for the GP with an estimated Sharpe ratio of more than double that of the MCI. However, the paper is not aiming at maximizing the Sharpe ratio. The resulting high Sharpe ratio is only a consequence of approximating the GP, which is at the efficient portfolio frontier.

Since the MCI seems unlikely to have the highest Sharpe ratio, many rulebased investment strategies have emerged. A large group of these strategies employs stock characteristics, known as factors, e.g. size, momentum or fundamental value, which have been found to have an effect on the expected returns of stocks; see, e.g. Rosenberg \& Marathe (1976). Fama \& French (1992), Carhart (1997) and Arnott et al. (2005). This raises the question about how sustainable some of these strategies are? If investors systematically exploit these factors, on a large scale, then the respective factor effects are likely to weaken or even vanish over time, as argued, e.g. in Van Dijk (2011). With the arbitrage pricing theory (APT), see Ross (1976), more complex multi-factor models were suggested than provided by the CAPM. The APT allows the systematic search for factors that may improve the performance of portfolios. The construction of the HWI exploits implicitly the presence of some factors that capture similarities in uncertainties driving stocks in the same industrial and geographical groupings. Contrary to most other approaches, the construction of the HWI avoids the explicit modeling or estimation of these factors. It only exploits their presence, which is unlikely to decline because common industrial and geographical uncertainties in similar economic activities are unlikely to vanish.

Many well-known optimal portfolio strategies aim at the minimum variance portfolio, as in Clarke et al. (2011); the risk parity portfolio, as in Maillard et al. (2010); the maximum diversification portfolio, as in Choueifaty \& Coignard (2008); or the hierarchical risk parity portfolio, as in Lopez de Prado (2016). These strategies employ estimated covariances of returns. As pointed out in Plyakha et al. (2014), the arising estimation errors easily offset the theoretical benefits of such optimal portfolio constructions. This is not even the biggest obstacle. More difficult than estimating covariances appears to be the accurate estimation of expected returns, which requires much longer observation windows than those usually available. This fact has been pointed out, e.g. in Best \& Grauer (1991), Chopra \& Ziemba (1993), Bai \& Ng (2002), Okhrin \& Schmid (2006), Ludvigson \& Ng (2007), Kan \& Zhou (2007), DeMiguel et al. (2009), Plyakha et al. (2014) and Kan et al. (2016). The difficulty in estimating drift parameters under any reasonable model seems to have 
hindered sample based portfolio optimization in becoming successfully implemented in practice.

\subsection{Diversification}

Despite decades of research on how to construct optimal portfolios, the simple, model-independent, equal-weighting approach seems to do at least as well as most other approaches suggested in the literature. This fact has been established in DeMiguel et al. (2009), where the authors show empirically that naive diversification - equal-weighting of stocks - outperforms sample-based mean-variance optimal portfolio construction. This paper explains these findings in Appendix A.3 via a Diversification Theorem, which states rather generally that well-diversified portfolios approximate asymptotically the GP for increasing number of stocks.

Let us intuitively explain the theoretical basis of the link between diversification and the GP. From now on we call the GP also our benchmark and stocks denominated in units of the GP benchmarked stocks. Purely for the purposes of this intuitive illustration, consider benchmarked stocks that have independent returns. From the theoretically given zero expected instantaneous returns of benchmarked stocks, see Theorem A.2, it follows that their returns, when formed over vanishing time periods, have zero mean. When constructing the simplest well-diversified index, the equally-weighted index (EWI) (starting with the same initial value as the GP), then the total return of the benchmarked EWI becomes the average of the independent returns of benchmarked stocks. By the Law of Large Numbers the total return of the benchmarked EWI converges to zero as the number of stocks increases. Consequently, the benchmarked EWI equals asymptotically the constant one for increasing number of stocks. By multiplying the limiting benchmarked EWI (the constant one) with the GP (denominated in units of the domestic currency) one obtains the GP in domestic currency. Thus, the EWI approximates asymptotically the GP for increasing number of stocks. The diversification theorem in Appendix C generalizes this convergence towards the GP for the HWI and many other welldiversified portfolios and is essentially an application of a generalized version of the Law of Large Numbers. The key observation is here that diversification brings portfolios close to the GP, which is a fundamental message that this paper conveys. The Diversification Theorem in Appendix A.3 generalizes this line of arguments and makes it applicable for the HWI and many other well-diversified portfolios.

To illustrate the effect of naive diversification in practice, we construct the equally-weighted index (EWI) for stocks of the developed markets and display the EWI in Fig. 1 together with the respective market capitalization weighted index (MCI). By constructing our new proxy for the GP, the hierarchically weighted index (HWI), we demonstrate in this paper that one can considerably improve (without estimation) the performance resulting from naive diversification by exploiting freely available information about similarities in uncertainties driving stock prices. Existing industrial and geographcial hierarchical classifications provide such 


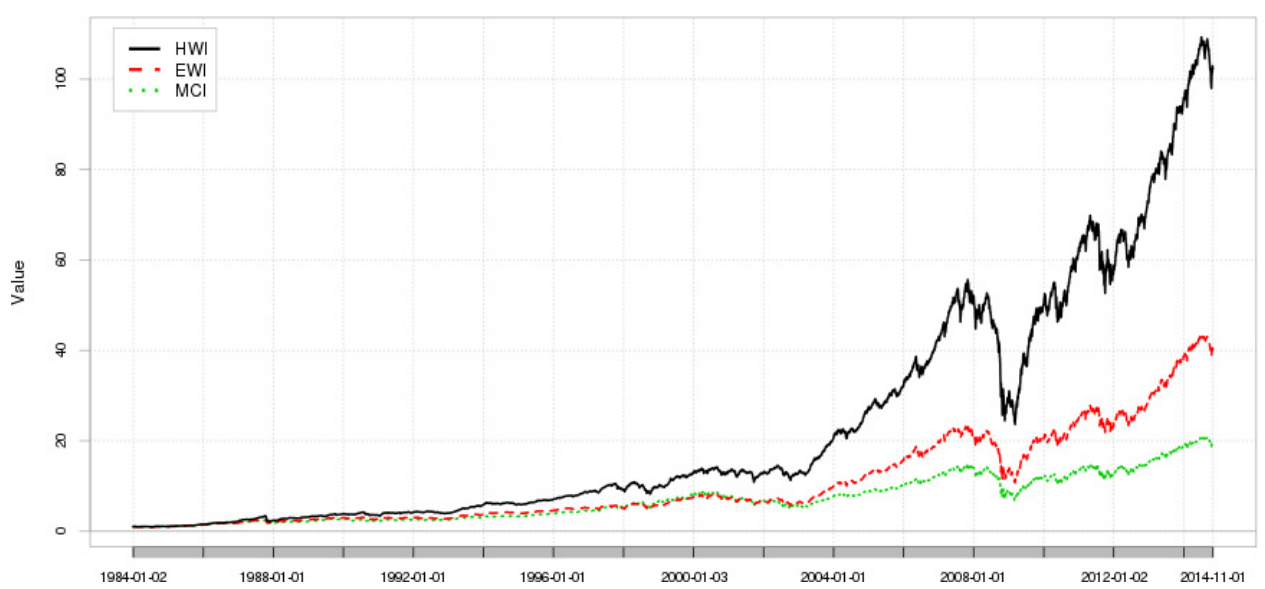

Fig. 1. Trajectories of HWI, EWI and MCI.

information. We use here the Industry Classification Benchmark (ICB); see Reuters (2008). Stocks that belong to the same industrial and geographical group are exposed to similar uncertainties plus their own specific uncertainty. Such hierarchical groupings remain stable over long periods of time. Most importantly, they persist also in periods of extreme market moves.

In Fig. 1 it seems that the EWI and the MCI were close to each other until 2004. The HWI appears to have outperformed the other two from the beginning. As the Diversification Theorem shows later on, both the EWI and the MCI converge towards the GP for increasing number of constituents. The EWI seems to have benefitted after 2004 more from the diversification effect than the MCI because it appears to be better diversified.

\subsection{Hierarchically weighted index as benchmark}

The HWI is constructed by, first, equal weighting the stocks within each industrial group in a country; second, equal weighting the resulting industry indexes in their country; third, equal weighting the respective country indexes in their region; and, finally, equal weighting the indexes of the regions. To illustrate the excellent performance of the HWI, we plot its trajectory along with those of the respective MCI and EWI for stocks of the developed markets in Fig. 1. All three indexes are denominated in US dollars and scaled so that they start with an initial value of one US dollar in January 1984.

Let $V_{t}^{\pi}$ denote the value at time $t$ of a strictly positive portfolio with strategy $\pi$, which in turn denotes the weights invested. We can then write the respective growth rate (GR) at time $t>0$ in the form

$$
G_{t}^{\pi}=\frac{1}{t} \ln \left(\frac{V_{t}^{\pi}}{V_{0}^{\pi}}\right) .
$$




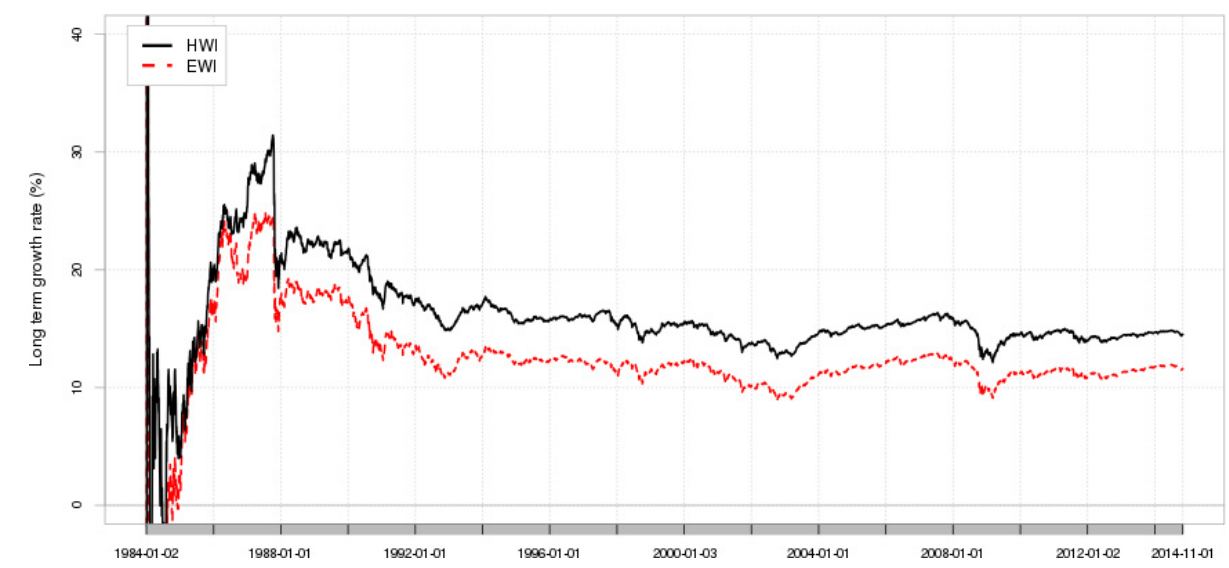

Fig. 2. Observed growth rates (GRs) in dependence on the final observation date for HWI and EWI.

The value of the GP at time $t \geq 0$ is denoted by $V_{t}^{\pi^{*}}$, where we set $V_{0}^{\pi^{*}}=1$. The GP is the portfolio that maximizes the expected GR $E\left(G_{t}^{\pi}\right)$ for all $t \geq 0$. We assume that $G_{t}^{\pi^{*}}<\infty$ almost surely (a.s.) for all $t \in[0, \infty]$.

In Fig. 2, we display the HWI's and the EWI's observed (annualized) GRs (in percent) as functions of the end date of the observation window. This figure shows that the GRs of the HWI and the EWI fluctuate similarly — a consequence of the fact that both well-diversified indexes are driven mostly by the nondiversifiable uncertainty of the stock market. This observation suggests to search for the "best" proxy for the GP among various competing, well-diversified portfolios by aiming for the largest GR of the longest available observation window. We note in Fig. 2 that the graphs fluctuate at the left end strongly. This is a consequence of the fact that we divide here by the short length of the respective observation window. For our longest available observation window the annualized GR in percent for the EWI is 11.6 and for the HWI 14.5, whereas the GR for the MCI reaches only 9.4; see also Table 3. After taking into account an average US interest rate of approximately $4.2 \%$ for the considered period, the GR of the discounted HWI turns out to be approximately double that of the discounted MCI.

This leads us to the important question - how close is the HWI to the true, hidden GP? The answer exploits the already mentioned fundamental fact that when using the true hidden GP as benchmark, all benchmarked stocks (with dividends reinvested) and all benchmarked self-financing portfolios have zero expected instantaneous returns; see Theorem A.2. This means that benchmarked portfolios are driftless and we call this the local martingale property of benchmarked securities. Since the local martingale property uniquely identifies the GP as the only portfolio for which this property holds, we can reject a candidate proxy for the GP if it generates significantly higher than zero expected instantaneous returns for stocks denominated in units of this proxy. 
By using the HWI as benchmark and averaging more than 30 million daily returns of benchmarked stocks, we show that the local martingale property cannot be rejected for the HWI. However, when taking the MCI, EWI and our other candidate proxies for the GP as a benchmark, then the local martingale property can be rejected.

With the HWI this paper does not only provide empirical evidence for the theoretically predicted local martingale property but reveals also the strict supermartingale property, where stocks, when denominated in units of the GP, form strict supermartingales and not true martingales. Additionally, it proposes with the HWI a new benchmark for equity fund management in developed markets that is extremely difficult to outperform.

The paper is organized as follows: In Sec. 2, we construct the HWI. Section3 analyzes the performance of the HWI, while Sec. 4 empirically establishes the local martingale property and the strict supermartingale property. Appendix A.1 describes the data, whereas Appendix A.2 proves properties of the GP. Appendix A.3 formulates and proves the Diversification Theorem.

\section{Hierarchically Weighted Index}

In this section, we introduce the hierarchically weighted index (HWI), which we implement for stocks in developed markets. Details on the Industry Classification Benchmark (ICB), see Reuters (2008), which we use when forming hierarchical groupings, together with information about the data, are provided in Appendix A.1.

\subsection{Portfolio construction}

We assume four levels in our hierarchy for stocks of the developed stock markets. This number of levels appears to be sufficient for achieving accurately enough the zero mean property for instantaneous returns of stocks of the developed markets when denominated in units of the HWI. At time $t \geq 0$ we denote by $M_{t}$ the number of geographical regions, $M_{t}^{j_{1}}$ the number of countries in the $j_{1}$ th region, $M_{t}^{j_{1}, j_{2}}$ the number of industrial groupings in the $j_{2}$ th country of the $j_{1}$ th region and $M_{t}^{j_{1}, j_{2}, j_{3}}$ the number of stocks in the $j_{3}$ th industrial grouping of the $j_{2}$ th country of the $j_{1}$ th region.

Let $S_{t}^{j}$ denote the cum-dividend price of the $j$ th stock (denominated in US dollars) at time $t \geq 0, j=\left(j_{1}, j_{2}, j_{3}, j_{4}\right)$. The portfolio weight for the investment in the $j$ th stock at time $t$ is denoted by $\pi_{t}^{j}$. The vector process of weights of a strictly positive, self-financing portfolio with value $V_{t}^{\pi}$ at time $t \geq 0$ is (with slight abuse of notation) denoted by $\pi=\left\{\pi_{t}=\left(\pi_{t}^{1}, \pi_{t}^{2}, \ldots, \pi_{t}^{N_{t}}\right)^{\top}, t \geq 0\right\}$, where

$$
N_{t}=\sum_{j_{1}=1}^{M_{t}} \sum_{j_{2}=1}^{M_{t}^{j_{1}}} \sum_{j_{3}=1}^{M_{t}^{j_{1}, j_{2}}} M_{t}^{j_{1}, j_{2}, j_{3}}
$$


denotes the number of stocks in our investment universe at time $t \geq 0$, and $x^{\top}$ the transpose of a vector or matrix $x$.

We introduce by $0=t_{0}<t_{1}<\cdots<t_{i}<t_{i+1}<\cdots$ the rebalancing times for a portfolio $V^{\pi}$. Its value $V_{t_{i}}^{\pi}$ at time $t_{i}$ is calculated, recursively, via the relation

$$
V_{t_{i}}^{\pi}=V_{t_{i-1}}^{\pi}\left(1+\sum_{j=1}^{N_{t_{i-1}}} \pi_{t_{i-1}}^{j} \frac{S_{t_{i}}^{j}-S_{t_{i-1}}^{j}}{S_{t_{i-1}}^{j}}\right)
$$

for $i \in\{1,2, \ldots\}$ with $V_{t_{0}}^{\pi}=1$. For all portfolios constructed in this paper the rebalancing frequency is quarterly. Additional rebalancing is performed when a stock "dies" between the quarterly rebalancing times. More frequent rebalancing, e.g. monthly, shows similar results to what we report.

The "traditional" benchmark for fund management is the market capitalization weighted index (MCI) with the weight $\pi_{t_{i}}^{\mathrm{MCI}, j}$ invested in the $j$ th stock at time $t_{i}$. This weight is determined by the reported respective market value $\mathrm{MV}_{t_{i}}^{j}$ via the formula

$$
\pi_{t_{i}}^{\mathrm{MCI}, j}=\frac{\mathrm{MV}_{t_{i}}^{j}}{\sum_{k=1}^{N_{t_{i}}} \mathrm{MV}_{t_{i}}^{k}}
$$

for $j \in\left\{1,2, \ldots, N_{t_{i}}\right\}, i \in\{0,1, \ldots\}$.

The weights $\pi_{t_{i}}^{E W I, j}$ for $j \in\left\{1,2, \ldots, N_{t_{i}}\right\}$ of the equally-weighted index (EWI) are set to be equal at each time $t_{i}$,

$$
\pi_{t_{i}}^{\mathrm{EWI}, j}=\frac{1}{N_{t_{i}}}
$$

for $i \in\{0,1, \ldots\}$.

\section{2. $H W I$}

The proposed hierarchically weighted index (HWI) uses industry, country and region groupings of stocks in a hierarchical manner to determine their respective weights. It invests equal fractions of wealth in the constituents of each group. Thus, the constituents of a group are themselves hierarchically weighted indexes, with the exception of the lowest level where they are stocks.

The three geographical regions we distinguish are Europe, Asia-Pacific and North-America. In the first column of Table 1, we list the 23 developed markets (countries) considered in this paper. These were chosen by using the ICB classification. The base dates for the start of investments made in each respective country are listed in column two. These are chosen by taking into account the reported number of dead stocks at a given time. We ignore Greece due to the downgrading of Greece to the status of an emerging market in 2013. We include Israel, since it was acknowledged as a developed market in 2010. 
Table 1. Base dates, number of stocks and type of industrial grouping used in a country for the construction of the HWI.

\begin{tabular}{lccl}
\hline Country & HWI country base date & No. of stocks & Industrial grouping \\
\hline Canada & $01 / 01 / 1990$ & 245 & Sector \\
United States & $02 / 01 / 1984$ & 998 & Subsector \\
Hong Kong & $01 / 01 / 1986$ & 127 & Sector \\
Japan & $01 / 01 / 1990$ & 1000 & Subsector \\
United Kingdom & $01 / 01 / 1985$ & 539 & Sector \\
Spain & $03 / 01 / 2000$ & 116 & Sector \\
Netherlands & $01 / 01 / 1990$ & 107 & Sector \\
Australia & $01 / 01 / 1988$ & 160 & Sector \\
Switzerland & $01 / 01 / 1992$ & 146 & Sector \\
Belgium & $02 / 01 / 1984$ & 90 & Sector \\
France & $01 / 01 / 1993$ & 247 & Sector \\
Germany & $01 / 01 / 1990$ & 235 & Sector \\
Italy & $01 / 01 / 1986$ & 150 & Sector \\
Singapore & $03 / 01 / 2005$ & 100 & Sector \\
Norway & $01 / 01 / 1990$ & 50 & Supersector \\
Ireland & $01 / 01 / 1991$ & 37 & Supersector \\
Sweden & $01 / 01 / 1991$ & 62 & Supersector \\
Finland & $01 / 01 / 1996$ & 47 & Supersector \\
Austria & $01 / 01 / 1992$ & 49 & Supersector \\
Portugal & $01 / 01 / 1993$ & 48 & Supersector \\
Denmark & $01 / 01 / 1993$ & 47 & Supersector \\
New Zealand & $03 / 01 / 2000$ & 50 & Supersector \\
Israel & $03 / 01 / 2000$ & 49 & Supersector \\
\hline
\end{tabular}

Due to these (and other) deviations of our set of stocks when compared to those of the Morgan Stanley Capital International Developed Markets Total Return Index (MSCI), we form the market capitalization weighted index (MCI), which allows us to make a fairer comparison with our constructed indexes. The constructed MCI deviates marginally from the MSCI, as we show later on. The total number of stocks considered is over 40000 . About 4810 stocks are selected dynamically at a given time from these stocks by the following rules, which make sure that, on average, we have a reasonable number of stocks in the industrial groups formed within each country: The rule for the selection of subsectors (in the ICB sense) as an industrial grouping, is that the country needs to have more than 900 stocks available. For countries with number of stocks between 80 and 900, we employ the sector grouping. For countries with fewer than 80 stocks the supersector grouping is used as an industrial grouping. For instance, in the case of the United States, we choose the 998 largest (by market value) stocks that are alive at a rebalancing date. The list of the number of stocks chosen for a given country is recorded in column three of Table 1 Column four in Table 1 indicates the level of ICB grouping we use as an industrial grouping of stocks in the given country.

In each group of the hierarchy we equal-weight the hierarchically weighted indexes we have formed at the next lower level. The weight for the $j$ th stock, with 
Table 2. Supersector and country weights for the HWI with comparison to the EWI and MCI.

\begin{tabular}{|c|c|c|c|c|c|c|c|}
\hline ICB Supersector & HWI & EWI & $\mathrm{MCI}$ & Country & HWI & EWI & MCI \\
\hline $\begin{array}{l}\text { Industrial goods \& } \\
\text { services }\end{array}$ & 13.95 & 16.19 & 11.42 & Canada & 16.67 & 5.219 & 4.243 \\
\hline $\begin{array}{l}\text { Personal \& household } \\
\text { goods }\end{array}$ & 6.889 & 4.729 & 5.272 & United States & 16.67 & 21.26 & 48.51 \\
\hline Real estate & 6.586 & 8.649 & 3.859 & Hong Kong & 6.667 & 2.706 & 4.499 \\
\hline Technology & 6.051 & 6.966 & 9.503 & Japan & 6.667 & 21.28 & 9.917 \\
\hline Retail & 5.932 & 5.944 & 5.492 & Australia & 6.667 & 3.409 & 2.819 \\
\hline Basic resources & 5.917 & 3.622 & 2.244 & Singapore & 6.667 & 2.13 & 1.27 \\
\hline Oil \& Gas & 5.482 & 6.221 & 8.978 & New Zealand & 6.667 & 1.065 & 0.1381 \\
\hline Food \& Beverage & 5.418 & 4.325 & 4.587 & United Kingdom & 2.083 & 11.48 & 7.865 \\
\hline Financial services & 5.146 & 7.861 & 4.23 & Spain & 2.083 & 2.45 & 1.807 \\
\hline Insurance & 5.033 & 2.897 & 4.137 & Netherlands & 2.083 & 2.28 & 1.238 \\
\hline Health care & 5.01 & 6.242 & 10.02 & Switzerland & 2.083 & 3.11 & 3.293 \\
\hline Utilities & 4.662 & 3.409 & 3.685 & Belgium & 2.083 & 1.917 & 0.8247 \\
\hline Telecommunications & 4.491 & 1.513 & 4.183 & France & 2.083 & 5.262 & 4.531 \\
\hline Travel \& Leisure & 4.393 & 4.325 & 3.029 & Germany & 2.083 & 4.985 & 3.753 \\
\hline $\begin{array}{l}\text { Construction \& } \\
\text { Materials }\end{array}$ & 3.624 & 4.154 & 1.434 & Italy & 2.083 & 3.174 & 1.397 \\
\hline Media & 3.237 & 2.983 & 2.6 & Norway & 2.083 & 1.065 & 0.6474 \\
\hline Chemicals & 3.107 & 2.812 & 2.699 & Ireland & 2.083 & 0.7882 & 0.1599 \\
\hline Banks & 2.956 & 4.708 & 9.696 & Sweden & 2.083 & 1.321 & 1.261 \\
\hline \multirow[t]{5}{*}{ Automobiles \& Parts } & 2.111 & 2.45 & 2.934 & Finland & 2.083 & 1.001 & 0.4259 \\
\hline & & & & Austria & 2.083 & 1.044 & 0.2295 \\
\hline & & & & Portugal & 2.083 & 1.001 & 0.1515 \\
\hline & & & & Denmark & 2.083 & 1.001 & 0.6733 \\
\hline & & & & Israel & 2.083 & 1.044 & 0.3431 \\
\hline
\end{tabular}

$j=\left(j_{1}, j_{2}, j_{3}, j_{4}\right)$, is then of the form

$$
\pi_{t}^{\mathrm{HWI}, j}=\frac{1}{M_{t}} \frac{1}{M_{t}^{j_{1}}} \frac{1}{M_{t}^{j_{1}, j_{2}}} \frac{1}{M_{t}^{j_{1}, j_{2}, j_{3}}}
$$

for $t \geq 0$. As a result, the weights of industrial groupings and countries in the HWI are rather different to those of the MCI and EWI, see Table 2, A visual illustration of the difference in the weights for the HWI and the MCI is given in Fig. 3. The stocks are here ordered by their market capitalization (taken at the end of our observation period) - starting with the stock with the largest market capitalization. The weights for the MCI are shown as black dashed curve and the respective weights for the HWI are included in red. The HWI employs information about stocks with exposure to similar uncertainties, whereas the EWI uses no such information.

\subsection{Other hierarchical groupings}

When forming the HWI we use the ICB's natural hierarchical grouping of stocks, starting with an industry grouping in a country, where the country is then part of a region. Well-diversified hierarchically weighted indexes can also be constructed 


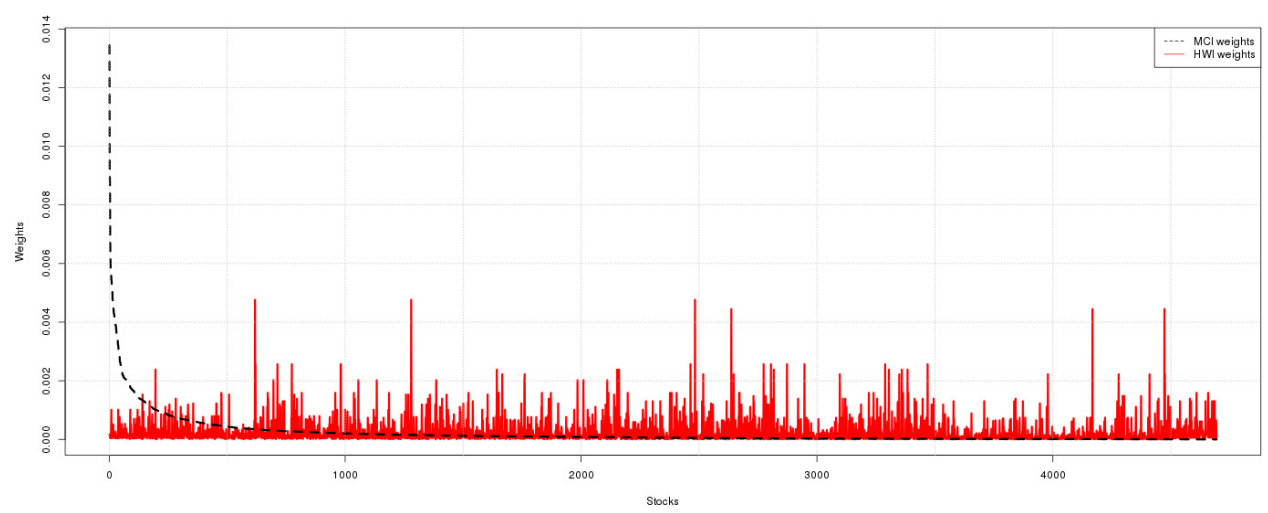

Fig. 3. The HWI and MCI weights for stocks ordered by market capitalization.

in other ways, e.g. by using only industrial or only geographical classifications of stocks. Furthermore, one may first use both geographical and industrial groupings at the lower levels of the hierarchy and at higher levels employ only industrial groupings, as illustrated in Platen \& Rendek (2012). This would still group stocks according to exposure to similar uncertainties and would provide well-diversified indexes.

An interesting question is - does it matter significantly if we tweak the groupings at the lower or higher levels of the hierarchy? As we will see below, the performance of an index with the natural industrial grouping at the lowest level and a geographical grouping at the second lowest level appears to be most favorable. However, the differences in the growth rates (GRs) are minor, as shown below.

To check whether there may be other, significantly better performing hierarchically diversified portfolios than the proposed HWI, we studied various alternative hierarchical groupings. We report the observed GRs for selected hierarchically weighted indexes and compare these to the HWI and EWI. First, we mention two illustrative examples of indexes that are diversified by their geographical origin at the stock level. These are the hierarchically weighted index diversified by region only (HWI.r) and the hierarchically weighted index diversified by country and then by region (HWI.c.r). The GRs (in percent) of the EWI and those of these two indexes are compared in Fig. 4 to the GR of the HWI. We observe that the HWI outperforms the HWI.c.r, which in-turn outperforms the HWI.r, with the EWI coming in last. These results indicate that the addition of an extra hierarchical level delivers an improvement in the GR. Note in Fig. 4 that the incorporation of some information about the region provides a marginal improvement over the performance of the EWI. Introducing the country level in the hierarchy enhances the GR the most. The GR improves further by introducing appropriate industrial groupings of stocks in each country, which is our proposed HWI. 


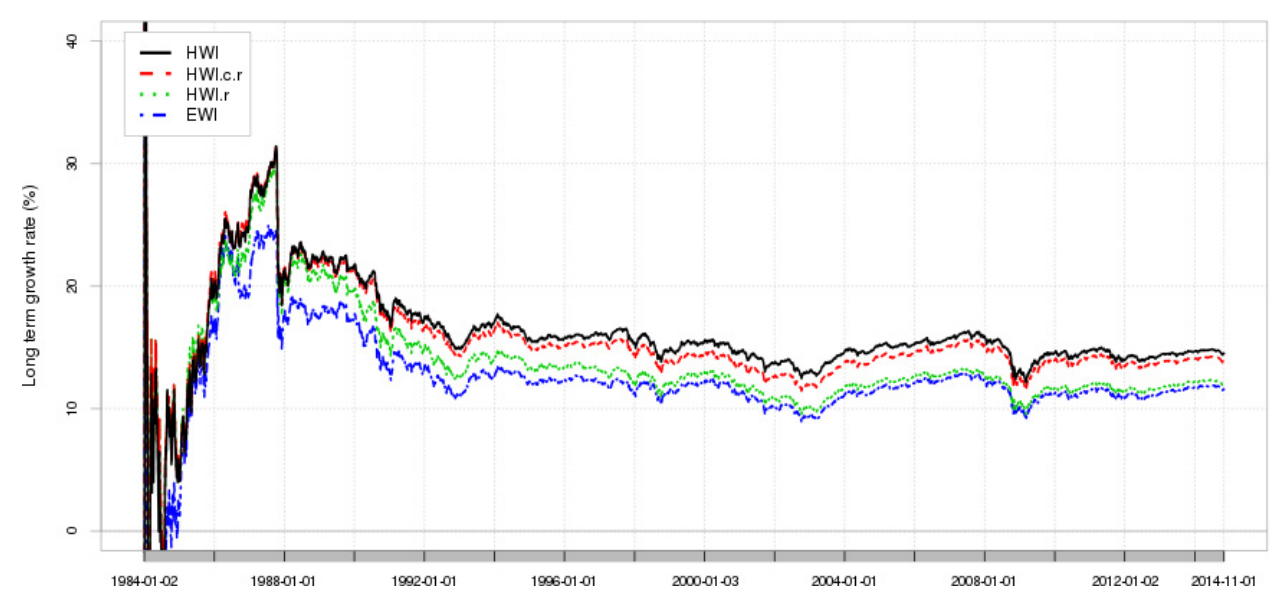

Fig. 4. GRs for the EWI, HWI.r, HWI.c.r and HWI.

We emphasize that the GRs of all these indexes fluctuate similarly, which already allows us to distinguish between their GRs after a few years of observation. The GRs for the first two years should be neglected in Fig. 4 because they display mainly noise that is enhanced by dividing by the short length of the observation period. The index with the highest GR at the end of the observation period is the HWI, as shown in Table 4 in the next section.

For further illustration, we mention three other examples of indexes grouped by their industrial origin on the stock level. These are: the hierarchically weighted index HWI.s diversified by global industry sector only, the hierarchically weighted index HWI.c.g diversified by country industry sector first and global industry sector second and the hierarchically weighted index HWI.c.r.g, diversified first by country sector, then by regional sector and, finally, by global sector. In comparison to these indexes and all similar indexes studied, the proposed HWI generates the highest observed GR for the longest available observation window.

\section{Further Empirical Results}

This section presents further empirical results concerning the performance of the HWI, EWI and MCI. In column two of Table 3 we report the observed (annualized) growth rate (GR) (in percent) over the longest available observation window of $T=$ 31 years, calculated according to formula (1.1). We emphasize that this has been our key performance measure so far in the current paper. It directly targets the GP when maximized. The traditionally used benchmark in equity fund management, the Morgan Stanley Capital International Developed Markets Total Return Index (MSCI), is included in Tables 36 for comparison. This index draws stocks from the same 23 developed markets we consider. However, the MSCI is constructed using approximately 1700 stocks, while the HWI, the MCI and our other indexes 
Table 3. Growth rate (GR), average return, risk premium, volatility and Sharpe ratio for the MSCI, MCI, EWI and HWI, respectively.

\begin{tabular}{cccccc}
\hline Index & GR & Average return & Risk premium & Volatility & Sharpe ratio \\
\hline MSCI & 9.23 & 10.48 & 6.26 & 15.79 & 0.3963 \\
MCI & 9.37 & 10.38 & 6.16 & 14.19 & 0.4343 \\
EWI & 11.58 & 12.33 & 8.11 & 12.20 & 0.6650 \\
HWI & 14.50 & 15.26 & 11.05 & 12.28 & 0.8997 \\
\hline
\end{tabular}

Table 4. Difference in average percentage growth rate over observation windows reaching from one to eight years with $95 \%$ confidence interval between HWI and MSCI, MCI, EWI, HWI.r and HWI.c.r, respectively.

\begin{tabular}{cccccc}
\hline Years & MSCI & MCI & EWI & HWI.r & HWI.c.r \\
\hline 1 & $5.41(5.22,5.61)$ & $5.21(5.04,5.39)$ & $2.78(2.68,2.88)$ & $2.53(2.43,2.63)$ & $0.62(0.59,0.66)$ \\
2 & $5.51(5.36,5.67)$ & $5.35(5.22,5.47)$ & $2.93(2.87,2.99)$ & $2.63(2.57,2.69)$ & $0.63(0.60,0.65)$ \\
3 & $5.63(5.49,5.76)$ & $5.35(5.24,5.46)$ & $3.00(2.96,3.04)$ & $2.65(2.61,2.69)$ & $0.62(0.60,0.65)$ \\
4 & $5.77(5.64,5.90)$ & $5.32(5.22,5.42)$ & $2.98(2.95,3.02)$ & $2.72(2.69,2.76)$ & $0.63(0.61,0.65)$ \\
5 & $5.91(5.79,6.03)$ & $5.30(5.20,5.39)$ & $3.01(2.98,3.03)$ & $2.81(2.78,2.83)$ & $0.64(0.62,0.66)$ \\
6 & $5.97(5.85,6.08)$ & $5.24(5.16,5.33)$ & $3.00(2.97,3.02)$ & $2.84(2.82,2.86)$ & $0.65(0.63,0.66)$ \\
7 & $5.96(5.85,6.07)$ & $5.17(5.09,5.25)$ & $2.99(2.96,3.01)$ & $2.87(2.84,2.89)$ & $0.66(0.64,0.68)$ \\
8 & $5.98(5.88,6.09)$ & $5.15(5.07,5.23)$ & $2.99(2.97,3.01)$ & $2.90(2.88,2.92)$ & $0.66(0.65,0.68)$ \\
\hline
\end{tabular}

Table 5. Relative frequency of outperforming the MCI over a given period length for the EWI and HWI.

\begin{tabular}{lcc}
\hline & EWI & HWI \\
\hline Daily & 0.526793 & 0.539724 \\
Monthly & 0.557688 & 0.605034 \\
Quarterly & 0.596067 & 0.654559 \\
Half-yearly & 0.618356 & 0.689812 \\
Yearly & 0.670045 & 0.747530 \\
2 Yearly & 0.748774 & 0.866499 \\
3 Yearly & 0.801810 & 0.885338 \\
5 Yearly & 0.777516 & 0.886695 \\
\hline
\end{tabular}

Table 6. Average relative drawdown and average recovery time (in days) for the MCI, EWI and HWI.

\begin{tabular}{lcc}
\hline Index & Av. Drawdown & Av. Recovery \\
\hline MCI & 0.0199 & 14.7533 \\
EWI & 0.0187 & 11.9556 \\
HWI & 0.0169 & 9.4541 \\
\hline
\end{tabular}

are constructed using over 4700 stocks at a time. Since the investment universe of the MSCI captures fewer sources of uncertainty than that of the MCI, one should expect a lower GR for the GP of the smaller investment universe available for the MSCI, which explains the slightly lower GR observed for the MSCI compared to that of the MCI. 
In Table 4 we show the average (with 95\% Gaussian confidence intervals) of the difference between the GRs of the HWI and those of the MSCI, MCI, EWI, HWI.r and HWI.c.r, respectively, estimated from all available observation windows of length 1-8 years, respectively. Note that the HWI performs best for all observation windows. Furthermore, we observe in Table 4 that the largest increase in the GR is achieved by the diversification over country indexes, that is, between HWI.r and HWI.c.r.

As we have seen in Fig. 1 and observe now also in Table 4 due to the almost parallel fluctuations of the well-diversified portfolios considered, it seems to take an observation window of only about one year to distinguish reasonably well between the GRs of the HWI, the MCI and the EWI. We emphasize that in Table 4 we can see that the difference between the GRs of the HWI and the MCI with the shortest window of one year is already at about $5 \%$ and it is statistically significant. For longer observation windows the respective confidence intervals become smaller but the mean remains about the same. Thus, systematic differences in the performance of different well-diversified portfolios can be reasonably accurately identified via the respective GRs of rather short observation windows. This is possible because the GP for the stocks of the developed markets turns out to be a well-diversified portfolio, as we confirm in the next section.

Despite our strategic focus on maximizing the GR, we also provide in the current section some popular short-term performance and risk measures by using the in equity fund management common definitions. The annualized percentage average returns are displayed in column three of Table 3 and the average risk premium is reported in column four. The average risk premium for the HWI is the highest and reaches approximately $11 \%$ compared to $6 \%$ for the MCI. The average annualized percentage volatility is recorded in column five, and equals $12 \%$ for the HWI, which is close to the average volatility for the EWI, whereas the average volatility for the MCI is higher and at about $14 \%$.

Figure 5 plots the average annualized daily return against the average annualized volatility for selected indexes, including some of those indexes with alternative hierarchical groupings mentioned in the previous section. The HWI exhibits the most favorable average annualized return, and has the highest Sharpe ratio, as shown in column six of Table 3. Furthermore, in Table 3, it can be seen that the improvement for the HWI in terms of its average return on the MCI, is about $4.9 \%$. This is above that for the EWI which amounts to $2.9 \%$. As mentioned in the introduction, the Sharpe ratio for the MCI is about 0.43, that for the EWI is 0.67 , and that for the HWI is about 0.90. The HWI exhibits the highest Sharpe ratio observed in our study. Theoretically, the GP is an efficient portfolio in the sense of Markowitz (1995), and exhibits the maximum Sharpe ratio. Since all portfolios at the efficient portfolio frontier have the same Sharpe ratio, one has to conclude that the MCI is unlikely to be positioned at the efficient portfolio frontier. Also, this observation seems to defy empirically assumptions typically made in a wide range of literature; see, e.g. Sharpe (1964). Table 5 shows observed relative frequencies for the event of 


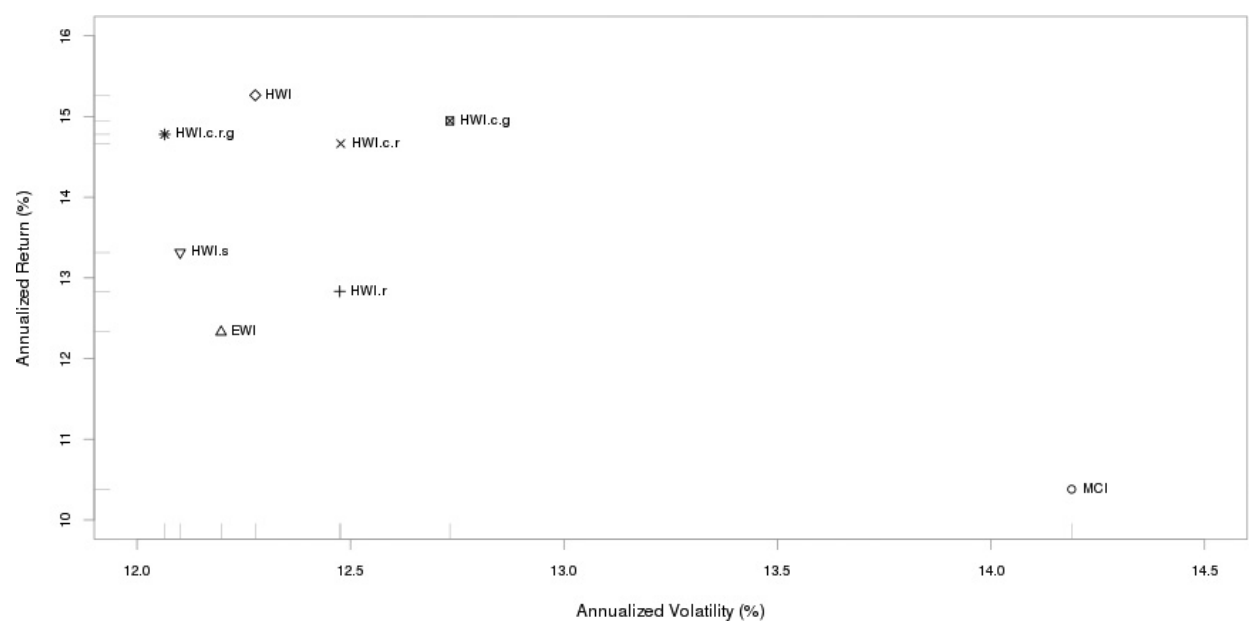

Fig. 5. Average return versus average volatility of selected indexes.

outperforming the MCI over a given period length. Note that the largest relative frequencies are observed for the HWI.

In Table 6, we summarize the average drawdown relative to the running maximum and the average time (in days) of recovery back to the level of the running maximum for the different indexes. Again, the HWI outperforms the MCI and the EWI. This is consistent with the theoretical prediction in Kardaras \& Platen (2010), where the GP is shown to require the shortest expected "market time" to reach a target level.

From the perspective of an equity fund manager, it is vital to address the impact of transaction costs for constructing and maintaining a portfolio. In Table 7 , we impose 40 basis points proportional transaction costs on every transaction, leading from the HWI, EWI and MCI to the HWI-TC, EWI-TC and MCI-TC, respectively. The turnover for the HWI is surprisingly low and as a result Table 7 shows only minor changes in performance estimates that should be compared to the respective values in Table 3. Most importantly, we observe only a minor decrease of the GR for the HWI from $14.50 \%$, shown in Table 3 , to the GR of the HWI-TC of $14.15 \%$, shown in Table 7 . This makes the HWI-TC a new benchmark for long-term investment securities that can be efficiently implemented in practice.

Table 7. Growth rate (GR) and other statistics for the HWI-TC, EWI-TC, MCITC constructed with 40 basis points proportional transaction costs.

\begin{tabular}{cccccc}
\hline Index & GR & Average return & Risk premium & Volatility & Sharpe ratio \\
\hline MCI-TC & 9.20 & 10.22 & 6.00 & 14.19 & 0.4228 \\
EWI-TC & 11.30 & 12.05 & 7.83 & 12.20 & 0.6423 \\
HWI-TC & 14.15 & 14.92 & 10.70 & 12.28 & 0.8714 \\
\hline
\end{tabular}


Note that for some institutional investors the above assumed proportional transaction costs may not even fully apply because the HWI strategy sells when stock prices increase and buys when stock prices decrease. In this manner the wider use of hierarchically weighted indexes like the HWI-TC provides highly desirable liquidity for the market. Moreover, this type of investment strategy stabilizes the market dynamics when widely implemented.

\section{Local Martingale Property}

We are now ready to empirically demonstrate that the local martingale property, see Theorem A.2 cannot be easily rejected for the hierarchically weighted index with transaction costs (HWI-TC) when used as a benchmark. This is a defining property for the GP. When the GP is used as benchmark, it causes expected instantaneous returns of benchmarked portfolios to be zero and, thus, never strictly positive. However, expected returns over strictly positive time periods can be negative. The latter property is a consequence of the, so called, supermartingale property of nonnegative benchmarked securities, which follows theoretically from the local martingale property; see Appendix A.2. Thus, one can reject (at a respective significance level) a candidate proxy for the GP by showing that the mean of returns for stocks benchmarked by this candidate proxy is strictly positive.

We gain strong evidence by combining all available daily returns of all benchmarked stocks in a large sample of more than thirty million (precisely 31, 472, 596) daily returns, where we employ the HWI-TC as benchmark. For comparison, we also employ the MCI-TC, EWI-TC, HWI.c.r-TC, HWI.c.g-TC and HWI.c.r.g-TC as benchmarks.

We consider all available daily, annualized percentage returns of all stocks (that constitute the HWI) when benchmarked by the index, shown in column one of Table 8, and display the respective sample mean in column two. One could argue that the returns are reasonably independent when observed on different days, and that the returns of different benchmarked stocks on the same day are also reasonably independent because they are mainly driven by their idiosyncratic (specific) uncertainties. Therefore, initially assuming independent and identically distributed returns, appears to be acceptable. The Central Limit Theorem determines then the length of the respective confidence intervals. In column three of Table 8 we show

Table 8. Test for the mean of daily annualized percentage returns of all benchmarked stocks.

\begin{tabular}{lcccrrr}
\hline Benchmark & Sample mean & Standard error & $99 \%$ LCI & $99 \%$ UCI & $Z$-test & $p$-value \\
\hline MCI-TC & 3.504079 & 0.142278 & 3.137594 & 3.870563 & 24.628 & 0 \\
EWI-TC & 0.936921 & 0.141376 & 0.572761 & 1.301081 & 6.627 & 0 \\
HWI-TC & -1.671584 & 0.141828 & -2.036909 & -1.306259 & -11.786 & 1 \\
HWI.c.r-TC & -1.072318 & 0.141815 & -1.437608 & -0.707027 & -7.561 & 1 \\
HWI.c.g-TC & -1.238168 & 0.141928 & -1.603750 & -0.872587 & -8.724 & 1 \\
HWI.c.r.g-TC & -1.364769 & 0.141681 & -1.729714 & -0.999823 & -9.633 & 1 \\
\hline
\end{tabular}


the resulting standard error and in columns four and five the lower level (LCI) and the upper level (UCI), respectively, of the $99 \%$ confidence interval for the "true" expected daily return of benchmarked stocks.

Some readers may be surprised to see several confidence intervals covering only negative values. However, this is a consequence of the theoretically possible strict supermartingale property of benchmarked stocks, a typical property of benchmarked securities under the benchmark approach. This property becomes here visible, since we are considering returns over the positive time period of one day and not instantaneous returns.

To make the above evidence even more precise, we capture it by using a standard test. Along this line, we denote by $\mu$ (in accordance with Theorem A.2 and A.11), the "true" expected average of all daily returns of all benchmarked stocks and test the hypothesis:

$$
H_{0}: \mu \leq 0 \text { versus } H_{1}: \mu>0 \text {. }
$$

We display the corresponding test statistic of the well-known Z-test (Mode 1966) in column six, and the respective one-sided $p$-values in column seven of Table 8 Using a $1 \%$ significance level, we can clearly reject $H_{0}$ for the MCI-TC and EWITC when used as benchmarks. However, when considering daily returns of benchmarked stocks we cannot reject $H_{0}$ for the HWI-TC, HWI.c.r-TC, HWI.c.g-TC and HWI.c.r.g-TC. This means that these hierarchically weighted indexes are closer to the GP than the MCI-TC and EWI-TC, and we need more evidence to decide which of the candidate proxies have to be rejected on the basis of a respective test.

One may argue that the assumption of independent and identically distributed returns in the first step of our analysis is too strong and should be relaxed. Therefore, in the second step of our study we remove this assumption and report in Table 9 the block bootstrap ${ }^{\text {a }}$ percentile $99 \%$ confidence intervals, with the respective test statistics and $p$-values for the hypothesis (4.1). We observe that the results in Table 9 resemble those in Table 8 .

Table 9. Bootstrap test for the mean of daily annualized percentage returns of benchmarked stocks.

\begin{tabular}{lcrrrr}
\hline Benchmark & Bootstrap mean & $99 \%$ LCI & $99 \%$ UCI & Test statistic & $p$-value \\
\hline MCI-TC & 3.502956 & 3.140459 & 3.910323 & 23.133 & 0 \\
EWI-TC & 0.936228 & 0.571123 & 1.398584 & 6.256 & 0 \\
HWI-TC & -1.664403 & -2.018709 & -1.277940 & -11.529 & 1 \\
HWI.c.r-TC & -1.074673 & -1.419532 & -0.674604 & -7.392 & 1 \\
HWI.c.g-TC & -1.243531 & -1.617380 & -0.893065 & -8.177 & 1 \\
HWI.c.r.g-TC & -1.361862 & -1.725689 & -1.001110 & -8.968 & 1 \\
\hline
\end{tabular}

a The block bootstrap replicates of the sample mean are obtained with the tsboot() function in the boot package in $R$, see, e.g. Davison \& Hinkley (2007), using block resampling with block lengths having a geometric distribution. 
Table 10. Test for the mean daily annualized percentage returns of HWI-TC benchmarked portfolios.

\begin{tabular}{lccccc}
\hline Benchmark & Sample mean & $99 \%$ LCI & $99 \%$ UCI & $Z$-test & $p$-value \\
\hline MCI-TC & -5.239304 & -8.707531 & -1.771077 & -3.89 & 1 \\
EWI-TC & -2.943474 & -4.914147 & -0.972799 & -3.85 & 1 \\
HWI.c.r-TC & -0.587549 & -1.219394 & 0.044296 & -2.4 & 0.99 \\
HWI.c.g-TC & -0.440414 & -2.217511 & 1.336683 & -0.64 & 0.74 \\
HWI.c.r.g-TC & -0.477921 & -1.354879 & 0.399037 & -1.4 & 0.92 \\
\hline
\end{tabular}

According to Theorem A.2, also the expected returns of nonnegative portfolios, when benchmarked by the GP, have theoretically the supermartingale property. Therefore, in Table 10, we test the average daily returns of the HWI-TC benchmarked MCI-TC, EWI-TC, HWI.c.r-TC, HWI.c.g-TC and HWI.c.r.g-TC according to hypothesis $H_{0}$ in (4.1). We note that this hypothesis cannot be rejected for the HWI-TC when used as a benchmark for each of the indexes considered in Table 10, Since all the $p$-values are above 0.7 , we also cannot reject for all these by the HWI-TC benchmarked portfolios the supermartingale property at the $1 \%$ level of significance. We repeated this study using the block bootstrap methodology and obtained very similar results. Thus, by using daily returns of benchmarked stocks and benchmarked candidate proxies for the GP, when the HWI-TC is used as benchmark, we cannot easily reject the supermartingale property.

It remains to ask - is it possible to reject on theoretical grounds some of the candidate proxies for the GP we constructed? To start with, we display in Fig. 6] the average returns of stocks, benchmarked by the HWI-TC, showing the $99 \%$

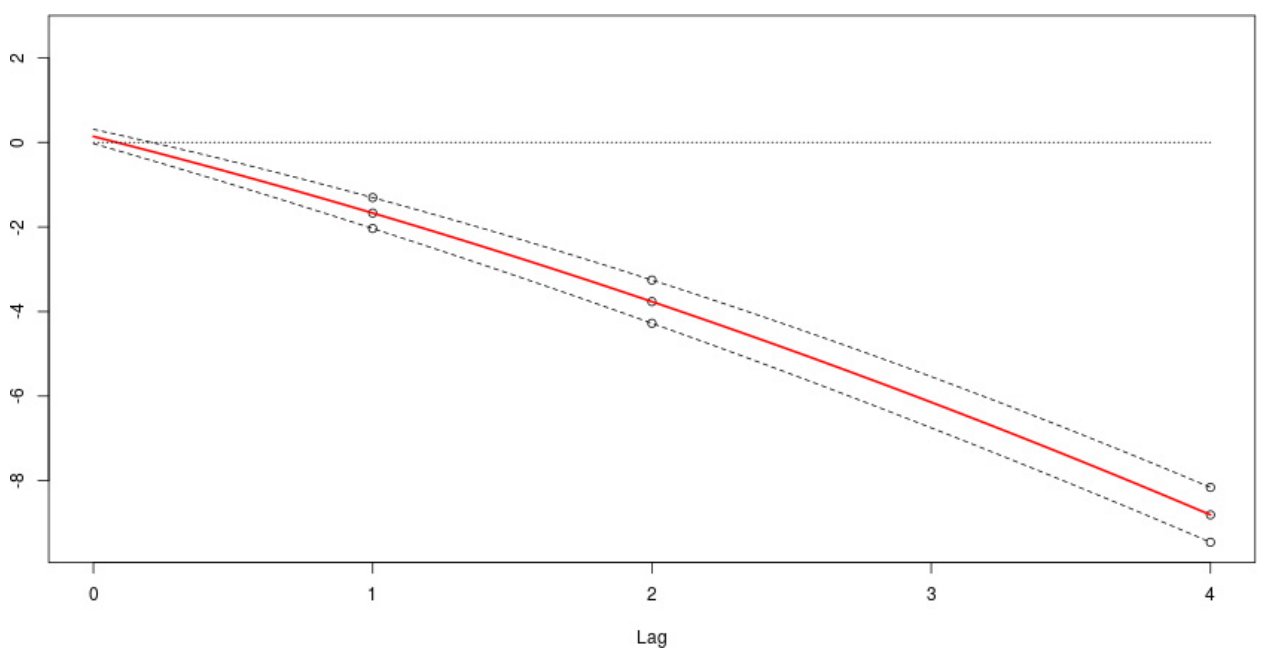

Fig. 6. Average annualized percentage returns of HWI-TC benchmarked stocks with $99 \%$ confidence intervals in dependence on the lag-time in days. 
confidence intervals when using all available daily, two-day and four-day returns, respectively. More precisely, let $\hat{S}_{t_{i}}^{j}$ denote the $j$ th by the HWI-TC benchmarked stock value at time $t_{i}$, which is the $i$ th day of our observations. Then the annualized percentage return of this security over the next following $k$ days equals $\hat{R}_{t_{i}}^{j}(k)=\left(\frac{\hat{S}_{t_{i+k}}^{j}}{\hat{S}_{t_{i}}^{j}}-1\right) 36500 / k$. We then average for each fixed lag $k=1,2,4$ the respective returns over all available stocks and all available time periods. In Fig. 6. the averages, together with the respective Gaussian $99 \%$ confidence intervals, are shown using circles for each of the three lags. This graphic visualizes clearly the strict supermartingale property of benchmarked stocks. The current benchmarked value of a stock is greater than its expected benchmarked one-day ahead value. For the returns of benchmarked stocks over two-day and four-day time periods, the average of the returns of benchmarked stocks becomes increasingly more negative with increasing length of the time period. Thus, when interpreting the HWI-TC as GP, Fig. 6 provides strong empirical evidence that benchmarked stocks are strict supermartingales and not true martingales. As explained in Heston et al. (2007) and Baldeaux et al. (2018), this strict supermartingale property indicates that stock price bubbles are present in developed markets.

We still would welcome evidence that would allow us to reject some of the remaining candidate proxies for the GP we have constructed. The local martingale property will give us the decisive tool. It refers to the theoretically predicted zero mean of instantaneous returns of benchmarked stocks when the GP is the benchmark. To check the local martingale property we need to see whether we cannot reject the property of zero expected instantaneous returns when using the HWI-TC as benchmark. To do this we extrapolate in Fig. 6] - via quadratic polynomials the averages, as well as, the upper and the lower bounds of the $99 \%$ confidence intervals. This gives us for the lag zero the extrapolated average instantaneous return with a value close to zero, which falls in Fig. 6] within the extrapolated $99 \%$ confidence interval at lag zero. For all other candidate proxies of the GP, which we constructed, the $99 \%$ confidence intervals for the mean of instantaneous returns of benchmarked stocks, when using these proxies as benchmark, cover only strictly positive values. This means, by modifying the previous $Z$-test for the mean of instantaneous returns of benchmarked stocks, the hypothesis $H_{0}$ in (4.1) has to be rejected for all candidate proxies for the GP as benchmarks with the exception of the HWI-TC.

Figure 6 shows that the average of instantaneous returns of stocks, when benchmarked by the HWI-TC, equals about $\hat{R}_{\mathrm{HWI}-\mathrm{TC}} \approx 0.002$, which is slightly above zero. When assuming the theoretically predicted local martingale property and using the hidden GP as benchmark, the average of instantaneous returns of stocks benchmarked by the GP would reach zero, that is $\hat{R}_{\mathrm{GP}} \approx 0$. Thus, with the hidden GP as benchmark one could potentially reduce the mean of instantaneous returns of benchmarked stocks by about

$$
\hat{R}_{\mathrm{HWI}-\mathrm{TC}}-\hat{R}_{\mathrm{GP}} \approx 0.002 .
$$


From stochastic calculus for diffusions we know that when denoting for benchmarked stocks the average squared volatility by $\sigma^{2}$, the average instantaneous growth rate by $\hat{G}$ and the average instantaneous return by $\hat{R}$, then $\hat{R}$ and $\hat{G}$ differ approximately by half the average squared volatility, that is

$$
\hat{R}-\hat{G} \approx \frac{1}{2} \sigma^{2}
$$

In column three of Table 8 , the averages of the volatilities of benchmarked stocks are obtained as square root of the average of respective squared volatilities. These averages of squared volatilities only range between $\sigma_{\mathrm{EWI}-\mathrm{TC}}^{2} \approx 0.02$ and $\sigma_{\mathrm{MCI}-\mathrm{TC}}^{2} \approx 0.0202$. This means, they do not depend much on the particular design of the as benchmark chosen well-diversified candidate proxy for the GP. Thus, we have approximately

$$
\hat{R}_{\mathrm{GP}}-\hat{G}_{\mathrm{GP}} \approx \hat{R}_{\mathrm{HWI}-\mathrm{TC}}-\hat{G}_{\mathrm{HWI}-\mathrm{TC}}
$$

Consequently, by (4.2) we get

$$
\hat{G}_{\mathrm{GP}} \approx \hat{G}_{\mathrm{HWI}-\mathrm{TC}}-\left(\hat{R}_{\mathrm{HWI}-\mathrm{TC}}-\hat{R}_{\mathrm{GP}}\right) \approx \hat{G}_{\mathrm{HWI}-\mathrm{TC}}-0.002 .
$$

Since the logarithm of a ratio equals the difference between the logarithms of the nominator and the denominator, we have $\hat{G}_{\mathrm{GP}} \approx g-G_{\mathrm{GP}}$ and $\hat{G}_{\mathrm{HWI}-\mathrm{TC}} \approx g-$ $G_{\mathrm{HWI}-\mathrm{TC}}$. Here $g, G_{\mathrm{GP}}$ and $G_{\mathrm{HWI}-\mathrm{TC}}$ can be interpreted as the average of the instantaneous growth rates of the in US dollar denominated stocks, GP and HWITC, respectively. Consequently, by (4.5) and for $G_{\mathrm{HWI}-\mathrm{TC}} \approx 0.1415$, see the GR of the HWI-TC in Table 7 , we obtain for the average of the instantaneous growth rate of the GP the estimate

$$
G_{\mathrm{GP}} \approx G_{\mathrm{HWI}-\mathrm{TC}}+0.002 \approx 0.1435
$$

This means that the potential increase in the GR by the GP, beyond the one we achieved already for the HWI-TC, can be only about $0.2 \%$. This does not leave much room for constructing improved proxies for the GP. Given the fact that we need no estimation for constructing the HWI-TC and that any further improvement would most likely involve the estimation of factors that may over time vanish because exploited, it seems not worthwhile to pursue this avenue.

One could only significantly increase the GR of the GP by increasing its investment universe. We note in Table 3 that the increase of the investment universe of the MSCI to the one used for the MCI gave a minor increase in the respective GR of about $0.14 \%$. Significantly larger increases are possible if one includes in the investment universe stocks of additional economies, in particular those of emerging markets. Preliminary studies indicate that a GR of about 18\% (and average volatility of about 11\%) seems to be realistic when using stocks of developed and emerging markets; see also Platen \& Rendek (2012). 


\section{Conclusion}

Due to the impossibility of obtaining sufficiently accurate estimates for means of returns, theoretically optimal stock portfolios are extremely difficult to construct in practice. This paper overcomes this long-standing problem by constructing a HWI, which approximates closely the GP for stocks of developed markets. Its construction does not rely on estimation. Instead, it diversifies by setting weights equal within groups of a natural industrial and geographical classification of stocks based on the respective underlying economic activities. The Diversification Theorem explains why the HWI and many other well-diversified portfolios approximate asymptotically the GP for increasing number of stocks. This convergence builds on the local martingale property of instantaneous returns of stocks and portfolios when denominated in units of the GP. This defining property of the GP cannot be easily rejected empirically for the HWI. Furthermore, it allows us to show that the by the HWI achieved approximation of the GP cannot be significantly improved. The access to an excellent proxy for the GP allows constructing other optimal portfolios with the GP as their central building block.

The paper provides strong empirical evidence that for stocks denominated in units of the HWI the current value appears to be strictly larger than their expected future values. This strict supermartingale property indicates the presence of stock price bubbles and demands a generalization of the classical no-arbitrage approach. Such generalization has been provided by the BA; see Platen \& Heath (2010). The GP is the central security under the BA with its existence as the only major requirement. The existence of an equivalent risk-neutral probability measure is not assumed, which allows employing a much wider modeling world than available under classical no-arbitrage assumptions. Some forms of classical arbitrage are permitted under the BA, including the existence of money market bubbles and stock price bubbles.

\section{Acknowledgments}

The authors would like to express their gratitude for receiving valuable suggestions on the research of the paper by two referees, Yacine Ait-Sahalia, Christa Cuchiero, Jerome Detemple, Vikram Kuriyan, Harry Markowitz, Jean-Charles Rochet, Erik Schloegl, David Taylor and Johan Walden, as well as, participants of workshops and conferences, including World Finance Conferences, Bachelier Finance Society World Congresses and Quantitative Methods in Finance Conferences. The Australian Research Council Grant DP130104074 supported the research.

\section{Appendix A}

\section{A.1 Data}

In this appendix, we describe the data employed. Thomson Reuters Datastream (TRD) provides a range of TRD calculated country, region and sector indexes 
Table A.1. Base dates and number of constituents for Datastream indexes.

\begin{tabular}{lrc}
\hline Country & Approx. no. of stocks & Base date \\
\hline Canada & 250 & Jan 1973 \\
United States & 1000 & Jan 1973 \\
Hong Kong & 130 & Jan 1973 \\
Japan & 1000 & Jan 1973 \\
United Kingdom & 550 & Jan 1965 \\
Spain & 120 & Jan 1986 \\
Netherlands & 130 & Jan 1973 \\
Australia & 160 & Jan 1973 \\
Switzerland & 150 & Jan 1973 \\
Belgium & 90 & Jan 1973 \\
France & 250 & Jan 1973 \\
Germany & 250 & Jan 1973 \\
Italy & 160 & Jan 1973 \\
Singapore & 100 & Jan 1973 \\
Norway & 50 & Jan 1980 \\
Ireland & 50 & Jan 1973 \\
Sweden & 70 & Jan 1982 \\
Finland & 50 & Mar 1988 \\
Austria & 50 & Jan 1973 \\
Portugal & 50 & Jan 1990 \\
Denmark & 50 & Jan 1973 \\
New Zealand & 50 & Jan 1988 \\
Israel & 50 & Jan 1992 \\
\hline
\end{tabular}

together with their current constituents and lists of dead stocks. For developed stock markets this paper builds global stock indexes from the data available in the TRD database. The 23 developed countries included in this study are given in the first column of Table A.1. These developed countries are identified based on the wellknown and widely used FTSE Industry Classification Benchmark (ICB) country classification; see Reuters (2008). For each of these developed markets Datastream uses a sample of stocks covering a minimum of $75-80 \%$ of total market capitalization by choosing the largest stocks by market value. Column two of Table A.1 lists the corresponding approximate number of stocks in the Datastream index for the selected 23 countries and in column three the relevant base date from which the index is available; see also Reuters (2008).

The respective list of constituents of Datastream country indexes was obtained from the TRD database by quoting the mnemonic for each country list. Table A.2 lists the mnemonics used for the active and dead stocks in the included markets. We also provide in this table the number of active and dead stocks presented on both mentioned lists for each country. Note that we only consider those stocks in the lists whose "GEOLN" = "GEOGN" = " Country Name". Additionally, companies with datatype "MAJOR" = "Y" are included, which means that for companies with more than one equity security the one with the largest market capitalization is chosen.

TRD classifies equities according to the previously mentioned Industry Classification Benchmark (ICB). We only consider those stocks that are classified into one 
Table A.2. Datastream stock lists and number of equity data obtained.

\begin{tabular}{lccccccc}
\hline Country & Active & No. active & Downl. & Dead & No. dead & Downl. & Removed \\
\hline Canada & LTOTMKCN & 250 & 245 & DEADCN1-2 & 6814 & 5310 & 598 \\
United States & LTOTMKUS & 999 & 998 & DEADUS1-6 & 22189 & 17009 & 467 \\
Hong Kong & LTOTMKHK & 130 & 127 & DEADHK & 248 & 200 & 8 \\
Japan & LTOTMKJP & 1000 & 1000 & DEADJP & 1681 & 1569 & 14 \\
United Kingdom & LTOTMKUK & 549 & 539 & DEADUK & 5625 & 5263 & 1037 \\
Spain & LTOTMKES & 120 & 116 & DEADES & 264 & 180 & 9 \\
Netherlands & LTOTMKNL & 117 & 107 & DEADNL & 429 & 343 & 39 \\
Australia & LTOTMKAU & 160 & 160 & DEADAU & 1784 & 1531 & 32 \\
Switzerland & LTOTMKSW & 150 & 146 & DEADSW & 360 & 246 & 12 \\
Belgium & LTOTMKBG & 90 & 90 & DEADBG & 271 & 245 & 23 \\
France & LTOTMKFR & 250 & 247 & DEADFR & 1534 & 1400 & 243 \\
Germany & LTOTMKBD & 250 & 235 & DEADBD & 3000 & 2229 & 21 \\
Italy & LTOTMKIT & 160 & 150 & DEADIT & 422 & 339 & 24 \\
Singapore & LTOTMKSG & 100 & 100 & DEADSG & 409 & 391 & 4 \\
Norway & LTOTMKNW & 50 & 50 & DEADNW & 415 & 400 & 34 \\
Ireland & LTOTMKIR & 37 & 37 & DEADIR & 129 & 108 & 22 \\
Sweden & LTOTMKSD & 70 & 62 & DEADSD & 819 & 709 & 72 \\
Finland & LTOTMKFN & 50 & 47 & DEADFN & 149 & 124 & 17 \\
Austria & LTOTMKOE & 50 & 49 & DEADOE & 196 & 160 & 8 \\
Portugal & LTOTMKPT & 50 & 48 & DEADPT & 239 & 165 & 51 \\
Denmark & LTOTMKDK & 50 & 47 & DEADDK & 277 & 254 & 15 \\
New Zealand & LTOTMKNZ & 50 & 50 & DEADNZ & 252 & 224 & 4 \\
Israel & LTOTMKIS & 50 & 49 & DEADIS & 505 & 413 & 1 \\
\hline & & & & & &
\end{tabular}

of the subsectors and exclude all stocks that are unclassified (UNCLAS) or classified as one of the following: unqouted equities (UQEQS), exchange traded funds (NEINV), suspended equities (SUSEQ) or other equities (OTHEQ). The number of such removed securities is recorded in the last column of Table A.2.

We use the ICB classification of stocks into subsectors, sectors and super- sectors, downloaded from Thomson Reuters Datastream (TRD) with mnemonics FTAG3, FTAG4, FTAG5. The country where the given stock originates is also recorded by TRD. Finally, the countries are grouped into three regions: Americas, Europe (EMEA) and Asia-Pacific.

The number of stocks for each active and dead list is recorded in Table A.2 in the fourth and seventh columns, respectively. For the downloaded stocks we obtained the total return prices and the market capitalization. The market value is understood to be the reported number of ordinary shares in the market multiplied by the stock price. Note that we have accounted for the fact that TRD repeats the last valid stock price or market capitalization for delisted stocks after a delisting. We found it necessary to remove this zero return from the end of the time-series. With the downloaded data prepared in this manner we perform our study, where we recover well the by TRD historically formed market capitalization weighted indexes for the 23 developed markets. We then use this data set for constructing the EWI, MCI and hierarchically weighted indexes. 


\section{A.2 Local martingale property}

\section{Market setting}

We prove in this appendix several theoretical results, which underpin the construction of well-diversified portfolios that approximate the GP. To avoid technicalities, we consider a continuous financial market. However, the key property we derive, the local martingale property, holds for most semimartingale markets; see Karatzas \& Kardaras (2007).

The traded or hedgeable uncertainty of stocks is modeled by an $n$-dimensional standard Brownian motion $W=\left\{W_{t}=\left(W_{t}^{1}, \ldots, W_{t}^{n}\right)^{\top}, t \in[0, \infty)\right\}, n \in\{2,3, \ldots\}$ on a filtered probability space $(\Omega, \mathcal{F}, \underline{\mathcal{F}}, P)$, satisfying the usual conditions; see, e.g. Karatzas \& Shreve (1998). Here, the filtration $\underline{\mathcal{F}}=\left(\mathcal{F}_{t}\right)_{t \geq 0}$ models the evolution of information generated by the Brownian motions driving the model. This information is characterized at time $t \geq 0$ by the sigma-algebra $\mathcal{F}_{t}$ and influences prices and other quantities constituting the market model. For matrices $x$ and $y$ we write $x \cdot y$ for the matrix product of $x$ and $y$. Recall, $x^{\top}$ denotes the transpose of $x$. Moreover, $\underline{1}=(1, \ldots, 1)^{\top}$ is a vector, and we write 0 for a zero matrix or vector, where the dimensions follow from the context.

Consider $m$ nonnegative, stocks with vector value process $S=\left\{S_{t}=\right.$ $\left.\left(S_{t}^{1}, \ldots, S_{t}^{m}\right)^{\top}, t \in[0, \infty)\right\}$, denominated in units of the domestic currency, which satisfies the (Itô-) stochastic differential equation (SDE)

$$
\frac{d S_{t}}{S_{t}}=a_{t} d t+b_{t} \cdot d W_{t}
$$

$t \in[0, \infty), S_{0}^{i}>0$ for $i=1, \ldots, m$. Note that all dividends are reinvested. The instantaneous expected return vector process $a=\left\{a_{t}=\left(a_{t}^{1}, \ldots, a_{t}^{m}\right)^{\top}, t \in[0, \infty)\right\}$ and the volatility matrix process $b=\left\{b_{t}=\left[b_{t}^{j, k}\right]_{j, k=1}^{m, n}, t \in(0, \infty]\right\}$ are assumed to be adapted and such that there exists a unique strong solution of the SDE (A.1); see, e.g. Sec. 7.7 in Platen \& Heath (2010) for respective sufficient conditions.

A strictly positive, self-financing, portfolio process $V^{\pi}$ is characterized by the weights or fractions of wealth $\pi_{t}=\left(\pi_{t}^{1}, \ldots, \pi_{t}^{m}\right)^{\top}, t \in[0, \infty)$, invested in the stocks, together with its positive initial value $V_{0}^{\pi}>0$, where

$$
\pi_{t}^{\top} \cdot \underline{1}=1
$$

The portfolio value $V_{t}^{\pi}$ at time $t$ then satisfies the SDE

$$
\frac{d V_{t}^{\pi}}{V_{t}^{\pi}}=\pi_{t}^{\top} \cdot \frac{d S_{t}}{S_{t}}=\pi_{t}^{\top} \cdot a_{t} d t+\pi_{t}^{\top} \cdot b_{t} \cdot d W_{t}
$$

for $t \in[0, \infty)$.

\section{Growth optimal portfolio}

We use interchangeably the notions of GP and expected logarithmic utility maximizing portfolio, also called Kelly portfolio, see Kelly (1956), even though there 
exist subtle differences, see Karatzas \& Kardaras (2007). We characterize the GP of the given continuous market by the following result, which follows directly from Theorem 3.1 in Filipovic \& Platen (2009).

Theorem A.1. If a GP exists in the given continuous market, then the process $\pi^{*}$ of GP weights may not be unique. However, the GP value process $V^{\pi_{*}}=\left\{V_{t}^{\pi_{*}}, t \in\right.$ $[0, \infty)\}$ is unique for some fixed initial portfolio value, which we set as $V_{0}^{\pi_{*}}=1$, and the SDE of the GP is of the form

$$
\frac{d V_{t}^{\pi_{*}}}{V_{t}^{\pi_{*}}}=\lambda_{t} d t+\theta_{t}^{\top} \cdot\left(\theta_{t} d t+d W_{t}\right)
$$

for $t \in[0, \infty)$. Here, we set

$$
\theta_{t}=b_{t}^{\top} \cdot \pi_{t}^{*}
$$

with $\pi_{t}^{*}$ and $\lambda_{t}$ representing the components of the solution of the equation

$$
\left(\begin{array}{cc}
b_{t} b_{t}^{\top} & 1 \\
\underline{1}^{\top} & 0
\end{array}\right)\left(\begin{array}{c}
\pi_{t}^{*} \\
\lambda_{t}
\end{array}\right)=\left(\begin{array}{c}
a_{t} \\
1
\end{array}\right)
$$

for all $t \in[0, \infty)$. A sufficient condition for the existence of a solution of (A.6) is the invertibility of the covariance matrix $b_{t} \cdot b_{t}^{\top}$ for all $t \in[0, \infty)$ together with the integrability of $\theta_{t}^{\top} \cdot \theta_{t}$. In the case when the risk-free asset is included in the investment universe, $\lambda_{t}$ equals its expected instantaneous rate of return and $\theta_{t}$ represents the vector of market prices of risk.

Assuming that the GP exists, it follows from the above theorem that the Lagrange multiplier $\lambda_{t}$ and the GP volatility vector $\theta_{t}$ are uniquely determined through $a_{t}$ and $b_{t}$. Moreover, by (A.5) and (A.6) the vector of expected instantaneous returns has the form

$$
a_{t}=\lambda_{t} \underline{1}+b_{t} \cdot \theta_{t} .
$$

Consequently, for any self-financing portfolio $V_{t}^{\pi}$, the SDE (A.3) takes the form

$$
\frac{d V_{t}^{\pi}}{V_{t}^{\pi}}=\lambda_{t} d t+\pi_{t}^{\top} \cdot b_{t} \cdot\left(\theta_{t} d t+d W_{t}\right) .
$$

\section{Local martingale property}

Under the BA, see Platen \& Heath (2010), the GP of a given set of constituents is called the benchmark. Any price process or portfolio $V_{t}^{\pi}$ is called benchmarked when denominated in units of the benchmark. By applying the Itô formula to the benchmarked portfolio value $\hat{V}_{t}^{\pi}=\frac{V_{t}^{\pi}}{V_{t}^{\pi_{*}}}$ we obtain from (A.8) and A.4 the SDE

$$
\frac{d \hat{V}_{t}^{\pi}}{\hat{V}_{t}^{\pi}}=\left(\pi_{t}^{\top} \cdot b_{t}-\theta_{t}^{\top}\right) \cdot d W_{t}
$$

for $t \in[0, \infty)$. The key observation is here that this SDE is driftless. Consequently, the following fundamental fact emerges: 
Theorem A.2 (Local martingale property). In a continuous market the expected instantaneous returns of self-financing portfolios, benchmarked by the GP, are equal to zero.

Since benchmarked portfolios satisfy driftless SDEs, they are, so called, local martingales. By Fatou's lemma any nonnegative local martingale is a supermartingale; see Platen \& Heath (2010). This means that any nonnegative benchmarked portfolio $\hat{V}^{\pi}$ satisfies the supermartingale property

$$
\hat{V}_{t}^{\pi} \geq E_{t}\left(\hat{V}_{t+h}^{\pi}\right)
$$

for all $0 \leq t \leq t+h<\infty$; see, e.g. Platen \& Heath (2010). Here, $E_{t}($.$) denotes$ the conditional expectation under the real-world probability measure $P$ given the information $\mathcal{F}_{t}$ at time $t$. The inequality (A.10) holds generally in semimartingale markets; see Karatzas \& Kardaras (2007). By the supermartingale property (A.10) we have for $0 \leq t \leq t+h<\infty$ the expected return

$$
E_{t}\left(\frac{\hat{V}_{t+h}^{\pi}-\hat{V}_{t}^{\pi}}{\hat{V}_{t}^{\pi}}\right) \leq 0,
$$

which yields the following statement:

Corollary A.2. There are only negative or zero but never strictly positive expected returns for nonnegative benchmarked securities.

Based on the above corollary, expected returns of benchmarked securities are never strictly positive. Consequently, a candidate for a proxy for the GP can be dismissed as an excellent proxy if when used as benchmark yields for benchmarked securities strictly positive expected returns. In this paper, we use this fact to identify the HWI-TC as an excellent proxy for the GP. By the local martingale property of benchmarked securities stated in Theorem A.2, one should expect the estimate for daily expected returns of an excellent proxy of the GP to be close to zero and, potentially, slightly negative. The respective estimated mean for instantaneous returns of benchmarked stocks should be about zero due to the local martingale property, which would identify the benchmark as an excellent proxy for the GP.

\section{A.3 Hierarchical diversification}

\section{Hierarchical grouping of stocks}

For approximating the GP better than is possible by naive diversification, we exploit naturally existing similarities in the uncertainties of economic activities for groups of companies. For instance, stocks of companies in a given industry can be expected to move largely together. Similarly, industries in a given country have common exposure to uncertainties that impact the respective economy. By forming a natural, hierarchical industrial and geographical grouping of stocks we are 
able to capture well enough these dependencies in the construction of the HWITC so that it becomes difficult to reject the HWI-TC as an excellent proxy for the GP.

Intuitively, we assume that factors exist that capture the similarities within the groups we form. However, we do not need to model or estimate these factors at any stage of the construction. It turns out to be sufficient to set weights equal within each group of stocks. The available large number of stocks and groups in the developed markets facilitates already in our study an excellent convergence towards the GP. As mentioned in the introduction, diversification is somehow the result of the convergence under a generalized Law of Large Numbers towards the mean of a sample, where the random variables are the returns of benchmarked stocks. Therefore, diversification does not require modeling or estimation. Diversification averages, essentially, the zero mean instantaneous returns of benchmarked stocks, yielding asymptotically zero total returns for benchmarked diversified portfolio. Assuming an initial value of 1.0 for the benchmarked diversified portfolio, it then follows that its value remains at the constant level 1.0. Consequently, the diversified portfolio is a proxy for the GP. This effect explains the fact that most well-diversified portfolios approximate the GP under rather general assumptions.

To formalize this insight in a Diversification Theorem, we make a few assumptions that help avoiding technicalities and allow to see the generality of hierarchical diversification approximating the GP. We assume that the stocks can be classified into hierarchical groupings with a fixed number $H \in\{1,2, \ldots\}$ of hierarchical levels - (for example, in the construction of the HWI we choose $H=4$ ). In the asymptotics captured by the Diversification Theorem we let the number $M \in\{2,3, \ldots\}$ tend to infinity. This number is approximately proportional to the number of constituents in a group. We emphasize, the number $H$ remains fixed. We assume that in each group of the hierarchy we have at least $\underline{K} M$ and at most $\bar{K} M$ next lower level subgroups, with fixed integers $\underline{K}$ and $\bar{K}, 0<\underline{K} \leq \bar{K}<\infty$. This means, for given $M$ we have at least $(\underline{K} M)^{H}$ and at most $(\bar{K} M)^{H}$ stocks in our investment universe.

To facilitate the existence of factors in our hierarchical stock market model, we denote by $W^{k_{1}}=\left\{W_{t}^{k_{1}}, t \in[0, \infty)\right\}$ the $k_{1}$ th independent, standard Brownian motion that primarily drives the $k_{1}$ th group on the highest level of the hierarchy, $k_{1} \in\{1,2, \ldots, \bar{K} M\}$. Furthermore, for $k_{1}, k_{2} \in\{1,2, \ldots, \bar{K} M\}$ we denote by $W^{k_{1}, k_{2}}=\left\{W_{t}^{k_{1}, k_{2}}, t \in[0, \infty)\right\}$ the $\left(k_{1}, k_{2}\right)$ th independent standard Brownian motion that primarily models the uncertainty driving the $k_{2}$ th group on the second highest level in the $k_{1}$ th group of the highest level. In an analogous manner, we introduce independent standard Brownian motions for next lower level groups until we reach at the lowest level the Brownian motions that drive specifically the stocks. Here $W^{k_{1}, k_{2}, \ldots, k_{H}}$ denotes the $\left(k_{1}, k_{2}, \ldots, k_{H}\right)$ th independent standard Brownian motion that primarily drives the $k_{H}$ th stock in the $k_{H-1}$ th lowest level group, of the $k_{H-2}$ th second lowest level group, etc. For the $j_{H}$ th benchmarked stock in the $j_{H-1}$ th lowest level group of the $j_{H-2}$-th second lowest level group, etc., we write 
$\hat{S}_{t}^{j}$, where $j=\left(j_{1}, j_{2}, \ldots, j_{H}\right) \in \Gamma_{M}=\{1,2, \ldots, \bar{K} M\}^{H}$. By using (A.1) and (A.7) we capture the hierarchical structure of the stock market dynamics for the $j$ th benchmarked stock price $\hat{S}_{t}^{j}$ by assuming for its dynamics the SDE

$$
\begin{aligned}
\frac{d \hat{S}_{t}^{j}}{\hat{S}_{t}^{j}}= & \sum_{k_{1}=1}^{\bar{K} M}\left(\psi_{t}^{j, k_{1}} d W_{t}^{k_{1}}+\sum_{k_{2}=1}^{\bar{K} M}\left(\psi_{t}^{j, k_{1}, k_{2}} d W_{t}^{k_{1}, k_{2}}\right.\right. \\
& \left.\left.+\cdots+\sum_{k_{H}=1}^{\bar{K} M} \psi_{t}^{j, k_{1}, k_{2}, \ldots, k_{H}} d W_{t}^{k_{1}, k_{2}, \ldots, k_{H}}\right)\right) .
\end{aligned}
$$

Note that any stock can be exposed to any of the uncertainties we model. It is the specification of the volatility processes that captures the dependence between stocks. By setting volatility coefficients to zero for some $j \in \Gamma_{M}$ we can easily model groups that have less than $\bar{K} M$ next lower level subgroups. Therefore, in our assumption on the number of members in a group this number is rather flexible. We emphasize that we do not need to model or estimate the specific dynamics of the volatility processes. The hierarchical structure of the above SDE turns out to be sufficient. What is important for the convergence is that the number $M$ is sufficiently large, which seems to be the case in our study.

The required minimal size for the number $M$ (to make the diversification theorem work) seems to be similar to the sample size needed to make the Law of Large Numbers work. Even when we do not have many members in some groups, the diversification effect seems to work reasonably well. As explained earlier in Sec. 2, we have chosen within a country the respective industry grouping in dependence on the number of stocks available in that country for constructing the HWI-TC.

\section{Diversification Theorem}

For given $M \in\{2,3, \ldots\}$ the SDE for a given benchmarked portfolio $\hat{V}_{t}^{\pi_{M}}$, with fraction $\pi_{M, t}^{j}$ invested in the $j$ th stock, $j \in \Gamma_{M}$, is by a.9 of the form

$$
\begin{aligned}
\frac{d \hat{V}_{t}^{\pi_{M}}}{\hat{V}_{t}^{\pi_{M}}}= & \sum_{j \in \Gamma_{M}} \pi_{M, t}^{j} \frac{d \hat{S}_{t}^{j}}{\hat{S}_{t}^{j}} \\
= & \sum_{k_{1}=1}^{\bar{K} M} \sum_{j \in \Gamma_{M}} \pi_{M, t}^{j} \psi_{t}^{j, k_{1}} d W_{t}^{k_{1}}+\sum_{k_{1}=1}^{\bar{K} M} \sum_{k_{2}=1}^{\bar{K} M} \sum_{j \in \Gamma_{M}} \pi_{M, t}^{j} \psi_{t}^{j, k_{1}, k_{2}} d W_{t}^{k_{1}, k_{2}} \\
& +\cdots+\sum_{k_{1}=1} \sum_{k_{2}=1}^{\bar{K} M} \ldots \sum_{k_{H}=1}^{\bar{K} M} \sum_{j \in \Gamma_{M}} \pi_{M, t}^{j} \psi_{t}^{j, k_{1}, k_{2}, \ldots, k_{H}} d W_{t}^{k_{1}, k_{2}, \ldots, k_{H}} .
\end{aligned}
$$

By the Itô formula, we obtain for the logarithm of the benchmarked portfolio $\hat{V}_{t}^{\pi_{M}}$ the SDE

$$
d \ln \left(\hat{V}_{t}^{\pi_{M}}\right)=\hat{g}_{t}^{\pi_{M}} d t+\frac{d \hat{V}_{t}^{\pi_{M}}}{\hat{V}_{t}^{\pi_{M}}}
$$


where $\hat{g}_{t}^{\pi_{M}}$ denotes the expected instantaneous growth rate of $\hat{V}_{t}^{\pi_{M}}$, see (A.18). Note that the benchmarked GP has trivially the constant value one. Therefore, its expected growth rate, the drift in the SDE of its logarithm, is zero. We identify good proxies for the GP as those for which at all times the expected instantaneous growth rate of their benchmarked value reaches asymptotically zero as $M$ tends to infinity. This means, we aim asymptotically for the maximum expected growth rate, the one of the GP.

Definition A.2. We call a sequence of benchmarked portfolio processes $\left(\hat{V}^{\pi_{M}}\right)_{M \in\{2,3, \ldots\}}$ a sequence of benchmarked approximate GP processes if

$$
\lim _{M \rightarrow \infty} \hat{g}_{t}^{\pi_{M}}=0
$$

almost surely for all $t \in[0, \infty)$.

For diversification to be possible, we need a condition that ensures that not all benchmarked stocks are driven to a significant extent by the same uncertainty. If this were the case, then this uncertainty would be most likely already captured by the nondiversifiable uncertainty driving the dynamics of the GP. Therefore, it is reasonable to make the following assumption.

Assumption A.2. For given $k_{1}, k_{2}, \ldots, k_{h} \in\{1,2, \ldots, \bar{K} M\}$ we assume that for all $M \in\{2,3, \ldots\}$ and all $h \in\{1,2, \ldots, H\}$

$$
\sum_{j \in \Gamma_{M}}\left|\psi_{t}^{j, k_{1}, k_{2}, \ldots, k_{h}}\right| \leq(\bar{K} M)^{H-h} \sigma_{t},
$$

where the adapted stochastic process $\sigma=\left\{\sigma_{t}, t \geq 0\right\}$ is almost surely finite for all $t \in[0, \infty)$.

Assumption A.2 covers an extremely wide range of stock market models. Note that the particular form of the volatilities $\psi_{t}^{j, \cdot}$ is not relevant. What is limited by (A.16) is the sum of the absolute values of the volatilities with respect to the same source of uncertainty. Under this assumption the convergence towards the GP can be secured by letting the weights for the stocks vanish "fast enough" for increasing $M$. We prove the following Diversification Theorem.

Theorem A.3 (Diversification Theorem). Under Assumption A.2, a sequence of benchmarked portfolios $\left(\hat{V}^{\pi_{M}}\right)_{M \in\{2,3, \ldots\}}$ is a sequence of benchmarked approximate GPs, if for each $M \in\{2,3, \ldots\}$ the maximum of the weights is limited by the relation

$$
\max _{j \in \Gamma_{M}}\left|\pi_{M, t}^{j}\right| \leq C M^{\xi-H}
$$

for some parameter $\xi \in\left[0, \frac{1}{2}\right)$, some constant $C \in(0, \infty)$ and all $t \in[0, \infty)$.

Obviously, relation (A.17) is satisfied for the EWI and the HWI, since for these indexes we have by (2.1), (2.4) and (2.5) that $\pi_{M, t}^{j} \leq \underline{K}^{-H} M^{-H}$ with $C=\underline{K}^{-H}$ 
and $\xi=0$ in A.17. Therefore, both indexes form sequences of approximate GPs. Since $\xi$ can be close to 0.5 , there are fractions allowed much larger than those for the stocks forming the EWI or HWI. The Diversification Theorem covers many MCIs, as long as, the number of stocks is large enough so that the asymptotics start to work. As we have seen, there typically exist much better proxies for the GP than the MCI. Still, the MCI is usually already a reasonable proxy for the GP. For larger stock markets it can be expected to become improved by hierarchical diversification because the GP accumulates in a hierarchically diversified manner the risk premia for the different sources of uncertainty in its SDE if these were tradeable.

\section{Proof of the Diversification Theorem A.3}

According to Definition A.2, we need to examine the expected instantaneous growth rate $\hat{g}_{t}^{\pi_{M}}$ of the benchmarked portfolio $\hat{V}^{\pi_{M}}$. By the Itô formula we obtain from Eqs. A.13) and (A.14) the expected growth rate in the form

$$
\begin{aligned}
\hat{g}_{t}^{\pi_{M}}= & -\frac{1}{2}\left(\sum_{k_{1}=1}^{\bar{K} M}\left(\sum_{j \in \Gamma_{M}} \pi_{M, t}^{j} \psi_{t}^{j, k_{1}}\right)^{2}+\sum_{k_{1}=1}^{\bar{K} M} \sum_{k_{2}=1}^{\bar{K} M}\left(\sum_{j \in \Gamma_{M}} \pi_{M, t}^{j} \psi_{t}^{j, k_{1}, k_{2}}\right)^{2}\right. \\
& \left.+\cdots+\sum_{k_{1}=1}^{\bar{K} M} \sum_{k_{2}=1}^{\bar{K} M} \cdots \sum_{k_{H}=1}^{\bar{K} M}\left(\sum_{j \in \Gamma_{M}} \pi_{M, t}^{j} \psi_{t}^{j, k_{1}, k_{2}, \ldots, k_{H}}\right)^{2}\right)
\end{aligned}
$$

for $t \in[0, \infty)$. By A.16) we obtain from that

$$
\begin{aligned}
\hat{g}_{t}^{\pi_{M} \geq} \geq & \frac{1}{2}\left(\max _{j \in \Gamma_{M}}\left|\pi_{M, t}^{j}\right|\right)^{2}\left(\sum_{k_{1}=1}^{\bar{K} M}\left(\sum_{j \in \Gamma_{M}}\left|\psi_{t}^{j, k_{1}}\right|\right)^{2}\right. \\
& +\sum_{k_{1}=1} \sum_{k_{2}=1}^{\bar{K} M}\left(\sum_{j \in \Gamma_{M}}\left|\psi_{t}^{j, k_{1}, k_{2}}\right|\right)^{2} \\
& \left.+\cdots+\sum_{k_{1}=1}^{\bar{K} M} \sum_{k_{2}=1}^{\bar{K} M} \ldots \sum_{k_{H}=1}^{\bar{K} M}\left(\sum_{j \in \Gamma_{M}}\left|\psi_{t}^{j, k_{1}, k_{2}, \ldots, k_{H}}\right|\right)^{2}\right) \\
\geq & -\frac{1}{2}\left(\max _{j \in \Gamma_{M}}\left|\pi_{M, t}^{j}\right|\right)^{2} \sigma_{t}^{2} \sum_{h=1}^{H}(\bar{K} M)^{h}\left((\bar{K} M)^{H-h}\right)^{2} \\
\geq & \left.-\frac{1}{2}\left(\max _{j \in \Gamma_{M}}\left|\pi_{M, t}^{j}\right|\right)^{2} \sigma_{t}^{2} \bar{K}^{2 H} \sum_{h=1}^{H}(M)^{h}(M)^{H-h}\right)^{2}
\end{aligned}
$$




$$
\begin{aligned}
& =-\frac{1}{2} \sigma_{t}^{2}\left(\max _{j \in \Gamma_{M}}\left|\pi_{M, t}^{j}\right|\right)^{2} \bar{K}^{2 H} M^{2 H-1} \sum_{h=1}^{H}\left(\frac{1}{M}\right)^{h-1} \\
& \geq-\frac{1}{2} \sigma_{t}^{2} \bar{K}^{2 H}\left(\max _{j \in \Gamma_{M}}\left|\pi_{M, t}^{j}\right|\right)^{2} \frac{M^{2 H-1}}{1-\frac{1}{M}} .
\end{aligned}
$$

The last estimate follows from the well-known formula for the limit of the sum of a geometric series. Thus, by using (A.17) we arrive at

$$
\begin{aligned}
\hat{g}_{t}^{\pi_{M}} & \geq-\frac{1}{2} C^{2} \sigma_{t}^{2} \bar{K}^{2 H} M^{2(\xi-H)} M^{2 H-1}\left(1-M^{-1}\right)^{-1} \\
& \geq-\frac{1}{2} C^{2} \sigma_{t}^{2} \bar{K}^{2 H} M^{2 \xi-1}\left(1-M^{-1}\right)^{-1} \\
& \geq-C^{2} \sigma_{t}^{2} \bar{K}^{2 H} M^{2 \xi-1} .
\end{aligned}
$$

Since $\xi \in\left[0, \frac{1}{2}\right)$, it follows almost surely for all $t \in[0, \infty)$ that

$$
\lim _{M \rightarrow \infty} \hat{g}_{t}^{\pi_{M}} \geq 0
$$

Since the GP has the highest expected instantaneous growth rate, the expected instantaneous growth rate of some benchmarked nonnegative security cannot be strictly positive, which by A.20 yields almost surely

$$
\lim _{M \rightarrow \infty} \hat{g}_{t}^{\pi_{M}}=0 .
$$

This proves by Definition A.2 the above Diversification Theorem.

\section{References}

R. Arnott, J. Hsu \& P. Moore (2005) Fundamental indexation, Financial Analyst Journal $61(2), 83-99$.

J. Bai \& S. Ng (2002) Determining the number of factors in approximate factor models, Econometrica 70 (1), 191-221.

J. Baldeaux, K. Ignatieva \& E. Platen (2018) Detecting money market bubbles, Journal of Banking and Finance 87, 369-379.

M. J. Best \& R. R. Grauer (1991) On the sensitivity of mean-variance-efficient portfolios to changes in asset means: Some analytical and computational results, Review of Financial Studies 4 (2), 315-342.

M. Carhart (1997) On persistence in mutual fund performance, Journal of Finance 52 (1), $57-82$.

V. K. Chopra \& W. Ziemba (1993) The effect of error in means, variances and covariances in optimal portfolio choice, Journal of Portfolio Management 19 (1), 6-11.

Y. Choueifaty \& Y. Coignard (2008) Towards maximum diversification, Journal of Portfolio Management 35 (1), 40-51.

R. Clarke, H. Silva \& S. Thorley (2011) Minimum-variance portfolio composition, Journal of Portfolio Management 37 (2), 31-45.

J. H. Cochrane (2001) Asset Pricing, Princeton University Press.

A. Davison \& D. Hinkley (2007) Bootstrap Methods and their Applications, Cambridge Series in Statistical and Probabilistic Mathematics. Cambridge University Press. 
F. Delbaen \& W. Schachermayer (1998) The fundamental theorem of asset pricing for unbounded stochastic processes, Mathematische Annalen 312, 215-250.

V. DeMiguel, L. Garlappi \& R. Uppal (2009) Optimal versus naive diversification: How inefficient is the 1/n portfolio strategy? Review of Financial Studies 22 (5), 19151953.

E. F. Fama \& K. R. French (1992) The cross-selection of expected stock returns, The Journal of Finance XLVII (2).

D. Filipovic \& E. Platen (2009) Consistent market extensions under the benchmark approach, Mathematical Finance 19 (1), 41-52.

C. Harvey, Y. Liu \& H. Zhu (2016) ... and the cross-section of expected returns, Review of Financial Studies 29 (1), 5-68.

S. L. Heston, M. Loewenstein \& G. A. Willard (2007) Options and bubbles, Review of Financial Studies 20 (2), 359-390.

R. Kan, X. Wang \& G. Zhou (2016) On the value of portfolio optimization in the presence of estimation risk: The case with and without risk-free asset, Working paper. University of Toronto.

R. Kan \& G. Zhou (2007) Optimal portfolio choice with parameter uncertainty, Journal of Financial and Quantitative Analysis 42, 621-656.

I. Karatzas \& C. Kardaras (2007) The numeraire portfolio in semimartingale financial models, Finance and Stochastics 11 (4), 447-493.

I. Karatzas \& S. E. Shreve (1998) Methods of Mathematical Finance, Springer.

C. Kardaras \& E. Platen (2010) Minimizing the expected market time to reach a certain wealth level, SIAM Journal of Financial Mathematics 1 (1), 16-29.

J. R. Kelly (1956) A new interpretation of information rate, Bell Systems Technology Journal 35, 917-926.

M. Loewenstein \& G. A. Willard (2000a) Local martingales, arbitrage, and viability: Free snacks and cheap thrills, Econometric Theory 16 (1), 135- 161.

M. Loewenstein \& G. A. Willard (2000b) Rational equilibrium asset-pricing bubbles in continuous trading models, Econometric Theory 91 (1), 17-58.

J. B. Long (1990) The numeraire portfolio, Journal Financial Economics 26, 29-69.

M. Lopez de Prado (2016) Building diversified portfolios that outperform out-of-sample, Journal of Portfolio Management 42 (4), 59-69.

S. Ludvigson \& S. Ng (2007) The empirical risk-return relation: A factor analysis approach, Journal of Financial Economics $\mathbf{8 3}$ (1), 171-222.

L. MacLean, E. Thorp \& W. Ziemba (2011) The Kelly Capital Growth Investment Criterion, World Scientific.

S. Maillard, T. Roncalli \& J. Teiletche (2010) The properties of equally weighted risk contribution portfolios, Journal of Portfolio Management 36 (4), 60-70.

H. Markowitz (1959) Portfolio Selection: Efficient Diversification of Investment, New York: Wiley.

R. C. Merton (1971) Optimum consumption and portfolio rules in a continuous-time model, Journal Economic Theory 3 (4), 373-413.

E. Mode (1966) Elements of Probability and Statistics. Englewood Cliffs, New Jersey: Prentice-Hall.

Y. Okhrin \& W. Schmid (2006) Distributional properties of portfolio weights, Journal of Economics 134, 235-256.

E. Platen (2002) Arbitrage in continuous complete markets, Advances in Applied Probability 34 (3), 540-558.

E. Platen \& D. Heath (2010) A Benchmark Approach to Quantitative Finance. Springer Finance, Springer. 
E. Platen \& R. Rendek (2012) Approximating the numeraire portfolio by naive diversification, Journal of Asset Management 13 (1), 34-50.

Y. Plyakha, R. Uppal \& G. Vilkov (2014) Equal or value weighting? Implications for asset pricing tests, Technical report, SSRN 1787045.

Reuters (2008) Datastream global equity indices, User Guide, Vol. 5, Thomson Reuters.

B. Rosenberg \& V. Marathe (1976) Common factors in security returns: Microeconomic determinants and macroeconomic correlates, University of California Institute of Business and Economic Research, Research Program in Finance, Working Paper No. 44.

S. A. Ross (1976) The arbitrage theory of capital asset pricing, Journal Economic Theory 13, 341-360.

W. F. Sharpe (1964) Capital asset prices: A theory of market equilibrium under conditions of risk, Journal of Finance 19, 425-442.

M. Van Dijk (2011) Is size dead? A review of the size effect in equity returns, Journal of Banking and Finance 35 (12), 3263-3274. 